\title{
PHASE SPACE UNIVERSALITY FOR MULTIMODAL MAPS
}

\author{
DANIEL SMANIA
}

\begin{abstract}
We study the dynamics of the renormalization operator for multimodal maps. In particular, we develop a combinatorial theory for certain kind of multimodal maps. We also prove that renormalizations of infinitely renormalizable multimodal maps with same bounded combinatorial type are exponentially close. Our results imply, for instance, the existence and uniqueness of periodic points for the renormalization operator with arbitrary combinatorial type.
\end{abstract}

\section{INTRODUCTION}

1.1. Multimodal maps. A multimodal map $f: I \rightarrow I, I=[-1,1]$, is a smooth map with a finite number of critical points, all of them local maximum or local minimum, and such that $f(\partial I) \subset \partial I$. Here we will be interested in more specific kinds of multimodal maps:

We say that $f$ is a multimodal map of type $n$ if it can be written as a composition of $n$ unimodal maps: to be more precise, if there exist maps $f_{1}, \ldots, f_{n}$ with the following properties

(1) $f_{i}: I \rightarrow I$ has an unique critical point (a maximum) and $f_{i}(\partial I) \subset \partial I$.

(2) $f=f_{n} \circ \cdots \circ f_{1}$.

(3) If $c_{i}$ is the critical point of $f_{i}$, then $f_{i}\left(c_{i}\right) \geq c_{i+1} \bmod n$.

The $n$-uple $\left(f_{1}, \ldots, f_{n}\right)$ is a decomposition of $f$. In this paper, we will assume that the unimodal maps are analytic and the critical points of $f_{i}$ are quadratic ( but observe that the critical points of $f$ are not, in general, quadratic). Clearly $f$ has many decompositions. Sometimes it is more convenient to decompose the dynamics of $f$ in its unimodal parts: for each decomposition of $f$ we can associate an extended map $F$ defined on $I_{n}=\{(x, i): x \in I, 1 \leq i \leq n\}$ (in other words, $I_{n}$ is a disjoint union of $n$ copies of $I$ ) by

$$
F(x, i)=\left(f_{i}(x), i+1 \bmod n\right)
$$

In [Sm1], we proved that deep renormalizations in infinitely renormalizable multimodal maps are multimodal maps of type $n$. This is the reason to restrict our attention for this kind of map.

We say that $J$ is a k-periodic interval to the extended map $F$ if

- $\left(c_{1}, 0\right) \in J\left(c_{i}\right.$ is the critical point of $\left.f_{i}\right)$,

- $\left\{J, F(J), \ldots, F^{k-1}(J)\right\}$ is an union of intervals with disjoint interior,

2000 Mathematics Subject Classification. Primary 37F25 37E20; Secondary 37F45.

Key words and phrases. renormalization, universality, multimodal, rigidity, hybrid class, tower.

This work has been partially supported by CNPQ 460110/00-4 and 0203/04-1, FAPERJ E26/151.896/2000 and FAPESP 2003/03107-9. I wish to thank W. de Melo for introducing me on this subject and for useful conversations about the mathematics and the style of this article. I am also grateful to IMPA, where this work was done. This paper is part of my thesis at IMPA. 
- The union of intervals in the above family contains $\left\{\left(c_{i}, i\right)\right\}$,

- $F^{k}(J) \subset J$, for $k>n$.

We will call $k$ the period of $J$.

Suppose that there exists a $k$-periodic interval for $F$. Let $P$ be the maximal interval which is a $k$-periodic interval for $F$. Then $F^{k}(\partial P) \subset \partial P$. We say that $P$ is a restrictive interval for $F$ of period $k$. Note that if $P$ and $\tilde{P}$ are, respectively, restrictive intervals for $F$ of period $k$ and $\tilde{k}, k<\tilde{k}$, then $\tilde{P} \subset P$. Let $P$ be a restrictive interval and let $0=\ell_{1}<\cdots<\ell_{n}$ be the times such that $\left(c_{i}, i\right) \in F^{\ell_{j}}(P)$ for some $i$. Let $P_{j}$ be the symmetrization of $F^{\ell_{j}}(P)$ in relation to $\left(c_{i}, i\right)$. Observe that $P_{j}$ contains a periodic point in its boundary. Let $A_{P_{j}}$ be the affine map which maps $P_{j}$ to $I$ and this periodic point to -1 . Then $g_{j}=A_{P_{j+1}} \circ F^{\ell_{j+1}-\ell_{j}} \circ A_{P_{j}}^{-1}$ is a unimodal map. Hence $g=A_{P_{1}} \circ F^{k} \circ A_{P_{1}}^{-1}$ is a multimodal map of type $n$ with decomposition $\left(g_{1}, \ldots, g_{n}\right)$. If $k>n$ is the minimal number such that $F$ admits a restrictive interval of period $k$, the map $g$ is called the renormalization of $f$, and denoted by $R(f)$. Indeed, it is easy to see that the definition of $R(f)$ does not depend on the decomposition.

The map $R(f)$ can be renormalizable again and so on. If this process can be continued indefinitely, we say that $f$ is infinitely renormalizable. Denote by $P_{0}^{k}$ the restrictive interval associated to the $k$-th renormalization $R^{k}(f)$. If $q \in C(F):=$ $\left\{\left(c_{i}, i\right)\right\}$, denote by the corresponding capital letter $Q_{0}^{k}$ the symmetrization of the interval $F^{\ell}\left(P_{0}^{k}\right)$ which contains $q$. We reserve the letter $p$ for $\left(c_{1}, 1\right)$. The critical point $r$ for $F$ will be the successor of the critical point $q$ at level $k$ if $r \in F^{\ell}\left(Q_{0}^{k}\right)$, for the minimal $\ell$ so that $F^{\ell}\left(Q_{0}^{k}\right)$ contains a critical point. Define $n_{r}^{k}=\ell$. Then, for any $r \in C(F), k \in \mathbb{N}$ and $i<n_{r}^{k}$, there exists an interval $R_{-i}^{k}$ so that

- $F^{i}$ is monotone in $R_{-i}^{k}$,

- $F^{i}\left(R_{-i}^{k}\right)=R_{0}^{k}$,

- The interval $F^{n_{r}^{k}-i}\left(Q_{0}^{k}\right)$ is contained in $R_{-i}^{k}$.

For details, see [Sm1].

Denote by $N_{k}$ the period of the restrictive interval $P_{0}^{k}$. We say that $f$ has C-bounded combinatorics if $N_{k+1} / N_{k} \leq C$.

For $(x, i),(y, j) \in I_{n}$, we say that $(x, i)<(y, j)$ if $i=j$ and $x<y$. The intervals of $I_{n}$ are the sets $J \times\{i\}$, for some $J \subset I$ and $i<n$. If $c_{i}$ is the critical point of $f_{i}$, denote $C(F)=\left\{\left(i, c_{i}\right)\right\}_{i}$.

Let $f$ and $g$ be two infinitely renormalizable multimodal maps of type $n$. We say that $f$ and $g$ have same combinatorics if $F^{i}\left(c_{k}\right)<F^{j}\left(c_{\ell}\right)$ if and only if $G^{i}\left(c_{k}\right)<G^{j}\left(c_{\ell}\right)$, for any $i, j \geq 0$ and $k$ and $\ell<n$, where $F$ and $G$ are unimodal decompositions of $f$ and $g$. For a domain $V \subset \mathbb{C}$, denote by $B(V)$ the Banach space of analytic functions defined in $V$ with a continuous extension to $\bar{V}$, provided with the sup norm.

Our main result is the following

Main Theorem 1. For $B \geq 2$, there exist a neighborhood $V \subset \mathbb{C}$ of $I$ and $\beta<1$ such that the following holds. If $f$ and $g$ are multimodal maps of type $n$, infinitely renormalizable and same combinatorics bounded by $B$, then, for $k \geq k_{0}(f, g)$, the $k$ th-renormalizations $R^{k} f$ and $R^{k} g$ have an analytic extension in $B(V)$ and

$$
\left\|R^{k} f-R^{k} g\right\|_{B(V)} \leq C \beta^{k}
$$

Here $C=C(f, g)$. 
The above result, together with another ones in this paper, imply the existence of periodic points to the renormalization operator with any wished combinatorics.

The renormalization theory has a long history: beginning with observable universality properties and conjectural explanation of these observations in families of unimodal maps, by Feigenbaum and Collet-Tresser. O. Lanford proposed the existence of a hyperbolic horseshoe to the renormalization operator. Similar conjectures was done for critical circle maps and for bimodal maps ([McKZ]).

A new step in the renormalization theory was attained by Sullivan's work $([\mathrm{Su}])$ : new tools was introduced, like quasiconformal deformation methods and a fruitful analogy with the theory of Kleinian groups. McMullen([McM2]) proved the exponential contraction of the renormalization operator and Lyubich ([L2]) its hyperbolicity (in the space of quadratic-like maps).

Our intention is to construct the foundation of the renormalization theory for multimodal maps. J. Hu (see [H1] and [H2]) studied the renormalization operator (compactness and contraction) for bimodal maps in the Epstein class. We will study the contraction of the renormalization operator in hybrid classes using the methods introduced by the cited authors for unimodal maps.

1.2. Outline of paper. In the section 1.1 we introduced the most important object in the paper: multimodal maps of type $n$. These maps are maps obtained of compositions of unimodal maps. Indeed, deep renormalizations of multimodal maps are multimodal maps of type $n$, so there are not loss of generality in restrict our study for these maps. Furthermore, these maps have a nice structure: in particular, we can define the combinatorial type of a renormalization, give explicit rules to the compositions of combinatorial types and realize any combinatorial type in sufficiently rich families. This is done in section 2 . In section 3 we study polynomials which are compositions of quadratic polynomials. In section 4 we introduce polynomial like maps of type $n$, and we prove that these maps are hybrid conjugated with compositions of quadratic polynomials. Moreover we study compact subsets in the space of polynomial like maps. In section 5 we define the complex version of renormalization and prove the 'small Julia set everywhere' theorem, which implies, in particular, that infinitely renormalizable polynomial like maps of type $n$ with bounded combinatorics does not support non trivial Beltrami fields in its Julia set. This result will be used in section 6 , where we prove that infinitely renormalizable real polynomials of type $n$ with same bounded combinatorial type are hybrid conjugated. As a corollary, we obtain that the set of infinitely renormalizable real polynomials of type $n$ with combinatorial type bounded by a constant $C$ is a Cantor set. In the section 7 we define the towers and use McMullen's arguments to prove it rigidity. Here we also prove that the critical points are deep points of the Julia set. The theory of towers is quite similar to the unimodal case and it implies the contraction of renormalization. Finally, in the section 7.2 we prove, using the McMullen's theory of dynamic inflexibility, the exponential contraction of the renormalization operator. In the appendix we collect some results about fixed-point theory, a special kind of Riemann surface and quasiconformal theory.

\section{Combinatorial Results}

Once we have defined infinitely renormalizable multimodal maps of type $n$, the natural question is how much freedom we have to built them, and if we can find them in arbitrary families of multimodal maps of type $n$. A satisfactory answer is 
given in section 2.1. But, first of all, we need to study the combinatorics of infinitely renormalizable maps. To be more precise, we will decompose the combinatorics of infinitely renormalizable multimodal maps of type $n$ in basic components, and show how to combine these basic components in an arbitrary way. If the reader is not interested in combinatorial aspects of the renormalization, we recommend to jump to section 3 .

Definition 2.1. Let $f$ be a multimodal map of type $n$. Let $\left(f_{1}, \ldots, f_{n}\right)$ be a decomposition of $f$. Let $x$ be a point in the domain of the extended map $F$ associated with this decomposition. The itinerary of $x$ with respect to the decomposition $\left(f_{1}, \ldots, f_{n}\right)$ is the infinity word $\ell_{0}(x) \ell_{1}(x) \ldots \ell_{i}(x) \ldots$, with $\ell_{i}(x)=L, C, R$ satisfying

$$
\ell_{i}(x)= \begin{cases}R & \text { if } F^{i}(x)>c_{j}, \text { for some } j ; \\ C & \text { if } F^{i}(x)=c_{j}, \text { for some } j ; \\ L & \text { if } F^{i}(x)<c_{j}, \text { for some } j .\end{cases}
$$

Let $(x, i)$ be a point of $I_{n}$. The inner itinerary of $(x, i)$ is the finite word $\ell_{0}(x, i) \ell_{2}(x, i) \ldots \ell_{n-i}(x, i)$.

Let $f$ be a multimodal map of type $n$. Order the critical points of $f, a_{1}<\ldots a_{k_{f}}$, $k_{f}<2^{n}$, and let $v_{1}, \ldots, v_{m}, m \leq n$ be the critical values of $f$. We associate to $f$ the structure $<k_{f}, \psi_{f}, \phi_{f}>$, where $\psi_{f}$ is the map of $\left\{i \in \mathbb{N}: 1 \leq i \leq k_{f}\right\}$ into $\{i \in \mathbb{N}: 1 \leq i \leq m\}$ such that $\psi_{f}(i)=j$ iff $f\left(a_{i}\right)=v_{j}$. Note that $\psi_{f}$ is defined up to a permutation in the critical values. The criticality of $f$ in $a_{i}$ is $\phi_{f}(i)$ (an even integer).

The definition of the structure $<k_{f}, \psi_{f}, \phi_{f}>$ does not depend on the decompositions, but the same is not obvious for the inner itinerary of the critical points of an extended map. However, as it is natural, these two combinatorial informations are completely equivalents.

Lemma 2.1. Let $f$ be a multimodal map of type $n$ with $n$. Then the inner itinerary of the critical points of the extended map $F$ depends only on the structure $<k_{f}, \psi_{f}, \phi_{f}>$. In particular, the inner itinerary does not depend on the decomposition of $f$. Furthermore, $<k_{f}, \psi_{f}, \phi_{f}>$ can be determined by the inner itinerary associated to a decomposition.

In an analogous way, the itinerary of an interval $J \subset I_{n}$ is defined by

$$
\ell_{i}(J)= \begin{cases}R & \text { if } F^{i}(J)>c_{j}, \text { for some } \mathrm{j} ; \\ C & \text { if } F^{i}(J) \text { contains } c_{j}, \text { for some } \mathrm{j} ; \\ L & \text { if } F^{i}(J)<c_{j}, \text { for some } \mathrm{j} .\end{cases}
$$

The inner itinerary of $J \subset I \times\{i\}$ is the finite word $\ell_{0}(J) \ldots \ell_{n-i}(J)$.

Corollary 2.1. Let $J$ be an interval in $I$, and $f$ a multimodal map of type $n$. Then the inner itinerary of $J$ does not depend on the decomposition.

By the above corollary, if $J$ is a $k$-periodic interval for some decomposition of $f$ then $J$ is $k$-periodic for all decomposition. In particular the maximal interval $P_{0}^{1}$ does not depend on the decomposition and so do the renormalization of $f$. Again by the previous theorem, the order of the intervals in the orbit of $J$ by an extended map $F$ in the $n$ copies on $I$ does not depend on the decomposition. 
Corollary 2.2. The itinerary of the point in $I \subset I_{n}$ with respect to an extended map $F$ does not depend on the decomposition.

The signal of a finite word $\omega=\ell_{0} \ldots \ell_{k}, \operatorname{sgn}(\omega)$, will be 1 , if there exists an even number of letters $R$ in $\omega$, or -1 otherwise. We will apply the signal function only on words which do not contain the letter $C$ (then we say that the word $\omega$ is pure). Provide the set of finite pure words with the following order $\prec$, defined by

- Provide the set of words with length one with the order: $L \prec C \prec R$.

- If $\omega=\ell_{0} \ldots \ell_{n}$ and $\tilde{\omega}=\tilde{\ell}_{0} \ldots \tilde{\ell}_{n}$ are such that $\ell_{0} \ldots \ell_{j}=\tilde{\ell}_{0} \ldots \tilde{\ell}_{j}$, but $\ell_{j+1} \neq \tilde{\ell}_{j+1}$, then

$-\omega \prec \tilde{\omega}$ if $\operatorname{sgn}\left(\ell_{0} \ldots \ell_{j}\right)=1$ and $\ell_{j+1}=L$; or $\operatorname{sgn}\left(\ell_{0} \ldots \ell_{j}\right)=-1$ and $\ell_{j+1}=R$.

- Otherwise $\tilde{\omega} \prec \omega$.

This is the usual order in the words with two symbols which occurs in the study of unimodal maps (see, e.g, $[\mathrm{dMvS}]$ ).

Lemma 2.2. Let $x, y \in I$. Assume that the pure itineraries $\ell_{0}(x) \ldots \ell_{j}(x)$ and $\ell_{0}(y) \ldots \ell_{j}(y)$ are distinct. Then $x<y$ if $\ell_{0}(x) \ldots \ell_{j}(x) \prec \ell_{0}(y) \ldots \ell_{j}(y)$.

Proof. The proof is easy.

Let $f$ and $\tilde{f}$ be multimodal maps of type $n$ with decompositions $\left(f_{1}, \ldots, f_{n}\right)$ and $\left(\tilde{f}_{1}, \ldots, \tilde{f}_{n}\right)$. If $c_{i}$ (resp. $\left.\tilde{c}_{i}\right)$ is the critical point of $f_{i}$ (resp. $\left.\tilde{f}_{i}\right)$, define $v_{i}=$ $f_{n} \circ \cdots \circ f_{i}\left(c_{i}\right)\left(\operatorname{resp} . \tilde{v}_{i}=\tilde{f}_{n} \circ \cdots \circ \tilde{f}_{i}\left(\tilde{c}_{i}\right)\right.$.

Lemma 2.3. Let $f$ and $\tilde{f}$ be multimodal maps of type $n$ with the same inner itinerary and such that $v_{i}<v_{j}$ iff $\tilde{v}_{i}<\tilde{v}_{j}$. Let $H_{0}, H_{1}: I \rightarrow I$ be increasing continuous functions such that $H_{1} \circ f=\tilde{f} \circ H_{0}$. Then, for $y \in I$ :

(1) $H_{0}\left(f^{-1}(y)\right)=\tilde{f}^{-1}\left(H_{1}(y)\right)$,

(2) For each pure word $\ell_{0} \cdots \ell_{n-1}$ there is at most one point $x \in f^{-1}(y)$ such that $\ell_{i}(x)=\ell_{i}$.

(3) There is a point $x \in f^{-1}(y)$ with inner itinerary $\ell_{0} \cdots \ell_{n-1}$ iff there is a point $\tilde{x} \in \tilde{f}^{-1}\left(H_{1}(y)\right)$ with the same itinerary. Furthermore $H_{0}(x)=\tilde{x}$.

Proof. The item 1 is obvious. To prove 2 , let $a_{1}<\cdots<a_{k}$ be the critical points of $f$ and $\tilde{a}_{1}<\cdots<\tilde{a}_{k}$ be the critical points of $\tilde{f}$. Notice that $H_{0}\left(a_{i}\right)=\tilde{a}_{i}$ and $H_{1}\left(v_{j}\right)=\tilde{v}_{j}$. By lemma 2.1 the inner itineraries of $c_{i}$ and $\tilde{c}_{i}$ are the same. Thus one gets that $f\left(a_{i}\right)=v_{j}$ iff $\tilde{f}\left(\tilde{a}_{i}\right)=\tilde{v}_{j}$. If the interval $\left[a_{i}, a_{i+1}\right]$ contains a preimage of $y$ then $v_{j}=f\left(a_{i}\right) \leq y \leq f\left(a_{i+1}\right)=v_{k}$. But this occurs iff $\tilde{v}_{j} \leq H_{1}(y) \leq \tilde{v}_{k}$ and so the interval $\left[\tilde{a}_{i}, \tilde{a}_{i+1}\right]$ contains a preimage of $H_{1}(y)$. Since the points in $\left(a_{i}, a_{i+1}\right)$ and $\left(\tilde{a}_{i}, \tilde{a}_{i+1}\right)$ have the same inner itinerary, the proof is finished.

Definition 2.2. Denote by $<A, \prec, A^{c}, \pi>$ the combinatorial data (c.d.) which contains

- A finite set $A$ with $A^{c} \subset A$. The set $A^{c}$ is the set of 'critical points' of $A$.

- $\prec$ is a transitive, anti-reflexive and anti-symmetric relation under $A$ such that any point in $A$ is comparable with an unique point in $A^{c}$. Furthermore, the relation 'a is comparable with $b$ on respect to $\prec$ ', $a \sim b$, is an equivalence relation. We will denote by $[x]$ the equivalence class of $x$ with respect to $\sim$.

- $\pi: A \rightarrow A$ is a map with the following property: if $c$ is a critical point in $A^{c}$ then 

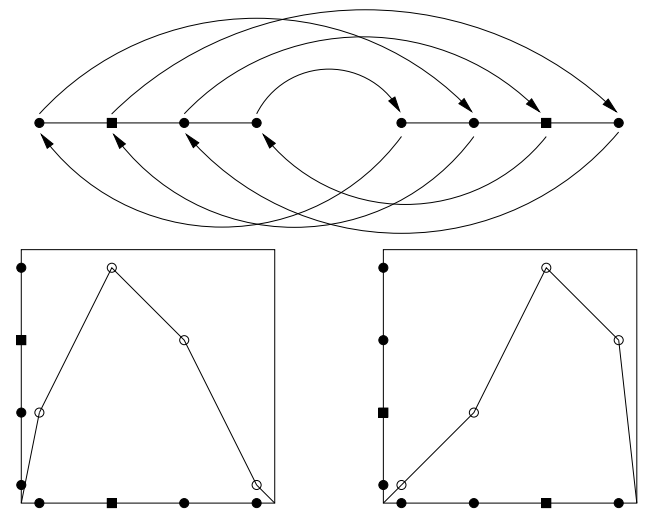

FiguRE 1. In the upper part of the figure we represented a c.d.: the elements of $A$ are represented by small discs and squares: the squares are the critical elements. Two elements of $A$ are comparable if they are in the same segment. In this case they respect the order in the real line. The elements are permuted as indicated by the arrows. In the bottom part we see that this c.d. can be realized by a multimodal map of type two.

- $a \prec b \prec c$ implies $\pi(a) \prec \pi(b) \prec \pi(c)$.

$-c \prec b \prec a$ implies $\pi(a) \prec \pi(b) \prec \pi(c)$.

- For any $a \in A$ there exists $c \in A^{c}$ so that $\pi^{i}(c)=a$, for some $i \geq 0$.

A marked combinatorial data (m.c.d.) will be $<A, \prec, A^{c}, \pi, c>$ with $c \in A^{c}$. Two m.c.d. $\langle\sigma, c\rangle,\langle\tilde{\sigma}, \tilde{c}\rangle$ will be identified up to bijections $\phi: A \rightarrow \tilde{A}$ satisfying

(1) $\phi\left(A^{c}\right)=\tilde{A}^{c}$

(2) For $x, y \in A, x \prec y$ iff $\phi(x) \tilde{\prec} \phi(y)$;

(3) $\pi=\phi^{-1} \circ \tilde{\pi} \circ \phi$ and

(4) $\phi(c)=\tilde{c}$.

Such map $\phi$ is called an isomorphism between m.c.d. Note that if $\sigma$ and $\tilde{\sigma}$ are two m.c.d. and $\pi$ is transitive (if $x, y \in A$ then $\pi^{i}(x)=y$, for some $i$ ) then there is at most one isomorphism between $\sigma$ and $\tilde{\sigma}$. A m.c.d. is essential if for any pair $a \prec b$ there is $i \geq 0$ so that $\pi^{i}(a) \preceq d \preceq \pi^{i}(b)$, for some $d \in A^{c}$. Clearly if any point in $A^{c}$ is periodic then $\sigma$ is essential.

Remark 2.1. We can associate two maps to a m.c.d. $\sigma$ : the first entry map to the critical set $\Pi: A \rightarrow A^{c}$ defined by $\Pi(x)=\pi^{i}(x)$, where $i \geq 0$ is minimal such that $\pi^{i}(x) \in A^{c}$; and the first return to the critical set, defined by $\Pi \circ \pi$.

Remark 2.2. Let $\sigma=<A, \prec, A^{c}, \pi>$ be a m.c.d. and $x \in A$. The itinerary of $x$ will be the periodic word $\ell_{0} \ell_{1} \ldots \ell_{n} \ldots$ satisfying

$$
\ell_{i}= \begin{cases}R & \text { if } \pi^{i}(x) \prec c, \text { for some } c \in A^{c} ; \\ C & \text { if } \pi^{i}(x) \in A^{c} ; \\ L & \text { if } c \prec \pi^{i}(x), \text { for some } c \in A^{c} .\end{cases}
$$

Definition 2.3. Let $f$ be a multimodal map of type $n$ and consider an extended map $F$ induced by a decomposition $\left(f_{1}, \ldots, f_{n}\right)$ of $F$. If $P$ is a periodic interval for $F$ of period $k$, then we can associate the following m.c.d. $\sigma=<A, \prec, A_{c}, \pi, c>$ 
- $A=\left\{F^{i}(P): 0 \leq i<k\right\}$.

- $A^{c}=\left\{F^{i}(P): c \in F^{i}(P)\right.$ for some critical point $c$ of $\left.F\right\}$. Moreover $c=$ $P$.

- For $F^{i}(P) \prec F^{j}(P)$, if $i=j \bmod n$ and $F^{i}(P)<F^{j}(P)$ in the usual order in the real line.

- $\pi: A \rightarrow A$ is defined by $\pi\left(F^{i}(P)\right)=F^{i+1 \bmod n}(P)$.

Note that $\pi$ is transitive. Furthermore the $\sigma$ does not depend on the decomposition (up to isomorphism between m.c.d). $\sigma=\sigma(P, f)$ will be called the combinatorial type of the periodic interval $P$. If $P$ is the restrictive interval of the renormalization $R f$ then $\sigma$ is the combinatorial type of the renormalization of $f$.

We can define in an analogous way the combinatorial type of a critically finite multimodal map.

Here it is easy to see why we must mark a critical point: for instance, let $f$ be a multimodal map of type 2 which is renormalizable and let $\left(f_{1}, f_{2}\right)$ be a decomposition of it. So the c.d associated to $f$ is a combinatorial information about the extended map $F: I_{2} \rightarrow I_{2}$ associated to this decomposition. If we mark one of the critical points we obtain the m.c.d. of $f_{1} \circ f_{2}$; if we mark the other critical point, we obtain the m.c.d. of $f_{2} \circ f_{1}$.

The set of $n$-admissible combinatorial types $\Sigma^{n}$ is the set of m.c.d. $\sigma$ which can be realized by critically finite multimodal map of type $n$ or the set of m.c.d. $\sigma$ such that $\#\left\{[x]: x \in A_{\sigma}\right\}=\# A_{\sigma}^{c}=n$. Denote by $\Sigma_{k}^{n}$ the subset of $n$-admissible transitive combinatorial types with period bounded by $k\left(\# A_{\sigma} \leq k n\right)$.

If $F$ is an extended map and $x$ is a point (or an interval) in $I_{n}$ with a pure itinerary $\omega=\ell_{0} \cdots \ell_{k}$, then $\operatorname{sgn}(\omega)$ says if $F^{k+1}$ preserves $(\operatorname{sgn}(\omega)=1)$ or reverses $(\operatorname{sgn}(\omega)=-1)$ the orientation in $x$. Let $\sigma=<A, \prec, A^{c}, \pi, c>$ be a transitive m.c.d. and $x \in A$. Let $i \geq 0$ be minimal so that $\pi^{-i}(x) \in \pi\left(A^{c}\right)$. Define $\operatorname{sgn}(\sigma)(x)=$ $\operatorname{sgn}\left(\ell_{0}\left(\pi^{-i}(x)\right) \cdots \ell_{i-1}\left(\pi^{-i}(x)\right)\right)$.

Definition 2.4. Let $\sigma_{1}=<A_{1}, \prec_{1}, A_{1}^{c}, \pi_{1}, c_{1}>, \sigma_{2}=<A_{2}, \prec_{2}, A_{2}^{c}, \pi_{2}, c_{2}>$ be m.c.d. such that $\pi_{1}$ is transitive and $\# A_{1}^{c}=\# A_{2}^{c}$. The product between $\sigma_{1}$ and $\sigma_{2}$ will be the m.c.d. $\sigma=\sigma_{2} * \sigma_{1}=<A, \prec, A^{c}, \pi, c>$ defined by

- $A=\left\{(x, y): \Pi_{1}(x)=\left(\Pi_{1} \circ \pi_{1}\right)^{i} c_{1}\right.$ and $y \in\left[\pi_{2}^{i} c_{2}\right]$, for some $\left.i\right\}$. Moreover $c=\left(c_{1}, c_{2}\right)$.

- $(x, y) \prec(\tilde{x}, \tilde{y})$ in the following cases:

$-x \prec_{1} \tilde{x}$

$-x=\tilde{x}, y \prec_{2} \tilde{y}$ and $\operatorname{sgn}\left(\sigma_{1}\right)(x)=1$;

$-x=\tilde{x}, \tilde{y} \prec_{2} y$ and $\operatorname{sgn}\left(\sigma_{1}\right)(x)=-1$.

- $A^{c}=\left\{(c, \tilde{c}) \in A: c \in A_{1}^{c}, \tilde{c} \in A_{2}^{c}\right\}$.

- $\pi$ is defined by

$$
\pi(x, y)= \begin{cases}\left(\pi_{1}(x), y\right) & \text { if } x \in A_{1} \backslash A_{1}^{c}, \\ \left(\pi_{1}(x), \pi_{2}(y)\right) & \text { if } x \in A_{1}^{c} .\end{cases}
$$

Note that $\# A^{c}=\# A_{1}^{c}=\# A_{2}^{c} . A$ m.c.d. $\sigma$ is primitive if $\sigma$ does not have a non trivial decomposition $\sigma=\sigma_{2} * \sigma_{1}$. Non trivial means \# $A_{1}^{c}<\# A_{1}$ and $\# A_{2}^{c}<\# A_{2}$

Remark 2.3. We are primarily interested in to define the $*$-product when $\pi_{1}$ is transitive, but we can give a more general definition when $\#\left\{(\Pi \circ \pi)^{i}\left(c_{1}\right): i \in \mathbb{N}\right\}=$ $\# A_{2}^{c}$. 
Proposition 2.1. The following holds:

- Let $f$ be a multimodal map of type $n$ with a periodic internal $P$. The combinatorial type of $P$ is primitive if and only if $P$ is the periodic interval associated with the first renormalization of $f$.

- Let $f$ be a renormalizable multimodal map of type $n$ which is $m$ times renormalizable and $R^{m} f=A_{P_{m}} \circ f^{N_{m}} \circ A_{P^{m}}^{-1}$. Then the order in the real line of the intervals of disjoint interior in the family $\left\{f^{j}\left(P^{m}\right): j<N_{m}\right\}$ are determined by $\sigma_{m} * \cdots * \sigma_{1}$, where $\sigma_{i}$ is the combinatorial type of the ith renormalization.

- Let $\sigma_{1}, \ldots, \sigma_{k}$ be an arbitrary sequence of primitive, transitive $n$-admissible m.c.d.. If $f$ is a critically finite multimodal map of type $n$ with combinatorial type $\sigma=\sigma_{k} * \cdots * \sigma_{1}$, then $f$ is $k$ times renormalizable, and the $(i-1)$ th renormalization of $f$ is critically finite with combinatorial type $\sigma_{i}$.

Proof. The arguments in the proofs of these three statements are quite similar. It is sufficient to prove that if $\sigma_{1}$ and $\sigma_{2}$ are $n$-admissible m.c.d., where $\sigma_{1}$ is transitive, then any critically finite multimodal map of type $n$ with combinatorial type $\sigma_{2} * \sigma_{1}$ has a restrictive interval $P$ of combinatorial type $\sigma_{1}$. Moreover, if $m$ is the period of $P$ then $f^{m}$ restrict to $P$ is critically finite with combinatorial type $\sigma_{2}$. Indeed, let $F$ be an extended map for $f$. Consider the representation of $\sigma$ given by the definition of $*$-product. Then $A_{\sigma} \subset\left\{(x, y): x \in A_{\sigma_{1}}\right.$ and $\left.y \in A_{\sigma_{2}}\right\}$. In other appropriate representation, $A_{\sigma}=\left\{F^{i} c: \mathrm{c}\right.$ is a critical point of $\left.F\right\}$. Let $\phi$ be the unique isomorphism between the first representation and the second one. For $x \in A_{1}$, let $P_{x}$ be the minimal interval in $I_{n}$ which contains all points in $\phi\left(\left\{(x, y):(x, y) \in A_{\sigma}\right\}\right)$. Then $P_{c_{1}}$ is a periodic interval for $f$ with combinatorial type $\sigma_{1}$. We leave the proof of that $f^{m}$ restricted to $P_{c_{1}}$ has combinatorial type $\sigma_{2}$ to the reader.

2.1. Full families. Now we will prove that, in quite natural families of multimodal maps, we can find infinitely renormalizable maps with arbitrary combinatorics. We will follow the steps of the generalization of the Milnor-Thurston theory for unimodal maps to multimodal maps introduced by de Melo and van Strien (see $[\mathrm{dMvS}])$. The main difference is that de Melo-van Strien deal with families where the number of critical points is constant. In our families. of multimodal maps (see below), the number of critical points goes of 1 to $2^{n}-1$.

Definition 2.5. A good family $f_{\lambda}$ of multimodal maps is a family of multimodal maps such that $f_{\lambda}=f_{n}(\lambda, \cdot) \circ \cdots \circ f_{1}(\lambda, \cdot)$, where the $C^{2}$-smooth functions $f_{i}: \Lambda \times$ $I \rightarrow I$, with $\Lambda \subset \mathbb{R}^{n}$, satisfies

- $f_{i}(\lambda, \cdot): I \rightarrow I$ is an unimodal map such that zero is its critical point. Moreover, zero is a non-flat critical point: around 0 , we can write $f_{i}=$ $\phi\left(|x|^{\ell_{i}}\right)$, where $\phi$ is a diffeomorphism and $\ell_{i}>1$.

- The function $\left(f_{1}(\cdot, 0), \ldots, f_{n}(\cdot, 0)\right): \Lambda \rightarrow I^{n}$ is a homeomorphism.

Let $\sigma=<A, \prec, A^{c}, c>$. Denote by $\lambda=\lambda\left(v_{1}, \ldots, v_{n}\right)$ be the parameter $\lambda \in \Lambda$ such that $\left(f_{1}(\lambda, 0), \ldots, f_{n}(\lambda, 0)\right)=\left(v_{1}, \ldots, v_{n}\right)$. Let $c_{1}=c$, and define inductively $c_{i+1} \in A^{c}$ as the unique critical point such that $c_{i+1} \in\left[\pi\left(c_{i}\right)\right]$. For $c_{i} \in[a]$ define

$$
f_{[a], \ell, \lambda}^{-1}=g_{i, \ell, \lambda}
$$


Here $\ell=R$ or $L$ and $g_{i, L, \lambda}$ (resp. $g_{i, R, \lambda}$ ) is the orientation preserving (resp. orientation reversing) inverse branch of $f_{i}(\lambda, \cdot)$. Consider the set

$K_{\sigma}=\left\{x \in[-1,1]^{A}:\right.$ if $a_{1}, a_{2} \in A$ and $a_{1} \prec a_{2}$ then $x_{a_{1}} \leq x_{a_{2}}$; if $\left.a \in A^{c}, x_{a}=0\right\}$

As a subset of $\mathbb{R}^{A}, K_{\sigma}$ is a compact convex set. Note that $x \in \partial K_{\sigma}$ iff $x_{a_{1}}=x_{a_{2}}$, with $\left[a_{1}\right]=\left[a_{2}\right], a_{1} \neq a_{2}$. Furthermore, if $x \in K_{\sigma}$

$$
\operatorname{dist}\left(x, \partial K_{\sigma}\right) \leq C d\left(x, \partial K_{\sigma}\right):=\min _{[a]=[b], a \neq b}\left|x_{a}-x_{b}\right|
$$

Here dist is the usual metric defined with a norm in $\mathbb{R}^{A}$. Define $T:$ int $K_{\sigma} \rightarrow$ int $K_{\sigma}$ as $T(x)=y$ where $y$ satisfies:

$$
y_{a}=f_{[a], \ell_{0}(a), \lambda}^{-1}\left(x_{\pi(a)}\right)
$$

Here $\lambda=\lambda\left(x_{\pi\left(c_{1}\right)}, \ldots, x_{\pi\left(c_{n}\right)}\right)$. The map $T$ is the Thurston map associated to the family $f_{\lambda}$.

Lemma 2.4. If $x \rightarrow \partial K_{\sigma}$ then

$$
\frac{|T(x)-x|}{\operatorname{dist}\left(x, \partial K_{\sigma}\right)} \rightarrow \infty
$$

Proof. The proof is exactly the proof of lemma 4.1 in pg. 126 of [dMvS]. We will omit the details. The argument is by contradiction. Assume that

$$
\frac{|T(x)-x|}{d\left(x, \partial K_{\sigma}\right)} \leq K
$$

Denote $y=T(x)$. Using the argument as in $[\mathrm{dMvS}]$, we can prove

(1) For $[a]=[b]$ :

$$
\left|x_{\pi(a)}-x_{\pi(b)}\right| \geq\left|y_{\pi(a)}-y_{\pi(b)}\right|-2 K d\left(x, \partial K_{\sigma}\right)
$$

(2) We also have, for $[a]=[b]$ :

$$
\left|x_{\pi(a)}-x_{\pi(b)}\right| \leq C\left|y_{a}-y_{b}\right|
$$

It follows

$$
\left|y_{\pi(a)}-y_{\pi(b)}\right| \leq C\left|y_{a}-y_{b}\right|+2 K d\left(x, \partial K_{\sigma}\right)
$$

Apply this inequality recursively to obtain

$$
\left|y_{\pi^{s}(a)}-y_{\pi^{s}(b)}\right| \leq C_{s}\left|y_{a}-y_{b}\right|+K_{s} d\left(x, \partial K_{\sigma}\right)
$$

Select $a$ and $b$ such that $d\left(y, \partial K_{\sigma}\right)=\left|y_{a}-y_{b}\right|$ and $s$ such that there is a critical point $c \in A^{c}$ such that $\pi^{s}\left(x_{a}\right) \preceq c \preceq \pi^{s}\left(x_{b}\right)$. Then

$$
C_{s} d\left(y, \partial K_{\sigma}\right) \geq\left|y_{c}-y_{\pi(a)}\right|-K_{s} d\left(x, \partial K_{\sigma}\right)
$$

Because $d\left(x, \partial K_{\sigma}\right), d\left(y, \partial K_{\sigma}\right) \rightarrow 0$ and since $y_{c}=0$ is a critical point to the extended map $F_{\lambda}$, one gets

$$
\frac{\left|y_{c}-y_{\pi(a)}\right|}{\left|x_{\pi(c)}-x_{\pi^{2}(a)}\right|} \rightarrow \infty
$$

But

$$
\frac{d\left(y, \partial K_{\sigma}\right)}{d\left(x, \partial K_{\sigma}\right)} \geq \frac{1}{C_{s}} \frac{\left|y_{c}-y_{\pi(a)}\right|}{\left|x_{\pi(c)}-x_{\pi^{2}(a)}\right|}-\frac{K_{s}}{C_{s}} \rightarrow \infty
$$

Which is a contradiction with (6).

Proposition 2.2. Let $\sigma$ be a n-admissible essential m.c.d.. Then any good family contains a critically finite multimodal map of type $n$ with combinatorial type $\sigma$. 
Proof. By de Melo-van Strien fixed point theorem (see the appendix), there exists a fixed point to the Thurston operator $T$ associated to a good family $f_{\lambda}$ and the essential combinatorial type $\sigma$.

Corollary 2.3. Let $f_{\lambda}$ be a good family. Given an infinity sequence of primitive, transitive m.c.d. $\left(\sigma_{1}, \sigma_{2}, \ldots\right)$, there exists $\lambda \in \Lambda$ such that $f_{\lambda}$ is an infinitely renormalizable map with this combinatorial type.

Proof. By Proposition 2.2, there exists a parameter $\lambda_{n}$ such that $f_{\lambda_{n}}$ is critically finite with combinatorics $\sigma_{n} * \sigma_{n-1} * \cdots * \sigma_{1}$. Let $n_{k}$ be a subsequence such that $\lambda_{n_{k}} \rightarrow \lambda_{\infty}$, for some $\lambda_{\infty} \in \Lambda$. Then $f_{\lambda_{\infty}}$ is infinitely renormalizable with combinatorics $\left(\sigma_{1}, \sigma_{2}, \ldots\right)$. Indeed, this is consequence of the following fact: for all $j>0$ there exist $\epsilon=\epsilon(j)>0$ and $n_{0}=n_{0}(j)$ such that $\left|f_{n}^{i}(0)\right| \geq \epsilon$, for $i \leq j, n>n_{0}$ (use that the critical point is non-flat).

\section{SpaCes of POLYNOMials}

3.1. Polynomials of type $\mathbf{n}$. Consider the polynomials of degree $2^{n}$ such that the dominant coefficient is 1 and 0 is a critical point of it. This space can be identified with the $\left(2^{n}-1\right)$-dimensional space $P_{n}$ of free coefficients. We say that $p \in P_{n}$ is a polynomial of type $\mathbf{n}$ if $p=P_{a_{n}} \circ \cdots \circ P_{a_{1}}$, with $P_{a}(z)=z^{2}+a$. Denote this set $\mathrm{Pol}_{n}$.

Proposition 3.1. Pol $_{n}$ is a complex submanifold of $P_{n}$ with global parameterization

$$
\left(a_{1}, \ldots, a_{n}\right) \rightarrow P_{a_{n}} \circ \cdots \circ P_{a_{1}}
$$

Proof. The following statement, proved by induction, is sufficient to prove the lemma: Let $\sum_{i \leq 2^{n}} b_{i} x^{i}=P_{a_{n}} \circ \cdots \circ P_{a_{1}}$ : if $i>2^{n}-2^{j}$ then $b_{i}=V_{i}\left(a_{1}, \ldots, a_{j-1}\right)$. If $i=2^{n}-2^{j}$ then $b_{i}=C_{j} a_{j}+V_{i}\left(a_{1}, \ldots, a_{j-1}\right)$, where $V_{i}$ are multi-variable polynomials and $C_{j} \neq 0$.

The connectivity locus of a family $f_{\lambda}, \lambda \in \Lambda$, of polynomial (or polynomiallike) maps is the set of parameters $\lambda$ such that the filled-in Julia set of $f_{\lambda}$ is connected. The following result are contained in the stronger results about centered monic polynomials proved by Branner and Hubbard $([\mathrm{BH}])$. But in our setting the proof is easy:

Proposition 3.2. The connectivity locus $\mathcal{C}_{n}$ of $\mathrm{Pol}_{n}$ is compact. Moreover all the connected filled-in Julia sets are contained in an uniform neighborhood of zero.

Proof. We claim that the connectivity locus is contained in the set $\left\{P_{a_{n}} \circ \cdots \circ\right.$ $\left.P_{a_{1}}:\left|a_{i}\right|<4\right\}$. Indeed, take a polynomial $F=P_{a_{n}} \circ \cdots \circ P_{a_{1}}$ in $A_{n}$ outside this set. Let $a_{M}$ such that $\left|a_{M}\right|=\max \left\{\left|a_{1}\right|, \ldots,\left|a_{n}\right|\right\}$. Consider a critical point $c$ such that $P_{a_{M}} \circ P_{a_{M-1}} \circ \cdots \circ P_{a_{1}}(c)=a_{M}$. We claim that $F^{n}(c)$ goes to infinity. This is consequence of a straightforward fact: if $b$ is such that $|b| \geq \max \left\{4,\left|a_{1}\right|, \ldots,\left|a_{n}\right|\right\}$ then $\left|b^{2}+a_{i}\right| \geq 2|b|$ for all $i$. Let $F=P_{a_{n}} \circ \cdots \circ P_{a_{1}}$ be a polynomial. Take $b$ like above. Then, using the fact above, $K(F) \subset B_{|b|}(0)$. In particular, in the connectivity locus the Julia sets are in a fixed neighborhood of zero. Now is easy to see that the connectivity locus is closed.

Proposition 3.3. For any $N>1$ there exists $\delta(N)$ with the following property: Consider $p \in \mathcal{C}_{n}$ and suppose that there exists $z$ such that $z, p^{N}(z) \in B_{\delta}$. Then $B_{2 \delta}$ is contained in a periodic component of $K(p)$ which contains a periodic attractor. 
Proof. Let $P=P_{a_{n}} \circ \cdots \circ P_{a_{1}}$ be a polynomial of type $n$ in $\mathcal{C}$. In particular $\left|a_{i}\right| \leq 4$. Then for any $\delta$ and $N$ there exists $C_{1}=C(N, \delta)$ such that $\left|D\left(p^{N}\right)(p(z))\right| \leq C_{1}$ for all $z \in B_{\delta}, p \in \mathcal{C}$. Furthermore there exists a constant $C_{2}=C_{2}(\delta)$ such that $\left|p^{\prime}(z)\right| \leq C_{2}|z|$. Suppose that $z_{0}, p^{N}\left(z_{0}\right) \in B_{\delta}$. For $z \in B_{2 \delta}$, we obtain

$$
\left|p^{N}(z)\right| \leq\left|p^{N}(z)-p^{N}\left(z_{0}\right)\right|+\left|p^{N}\left(z_{0}\right)\right| \leq\left(2 C_{1}(2 \delta, N-1) C_{2}(2 \delta) \delta+1\right) \delta
$$

Thus if $\delta$ is small enough, then the map $p^{N}: B_{2 \delta} \rightarrow B_{2 \delta}$ is a strict contraction, and the lemma follows.

Corollary 3.1. There exists a constant $C$ so that for any $p \in \mathcal{C}_{n}$

$$
1 / C \leq \operatorname{diam} K(p) \leq C .
$$

\section{Polynomial LiKe MAPS}

We say that $f: U \rightarrow V$, where $U$ and $V$ are simply connected domains such that $U$ is compactly contained in $V$, is a polynomial like map if $f$ is a holomorphic ramified covering. The filled-in Julia set $K(f)$ of $f$ is the set of points in $U$ for which all iterates of $f$ are defined. We assume that the McMullen's topology in the space of polynomial-like maps (see [McM1]) is familiar to the reader. Sometimes it is useful work with germs of polynomial like maps: two polynomial like maps $f_{i}: U_{i} \rightarrow V_{i}, i=1,2$ define the same germ if

- The filled-in Julia sets $K\left(f_{1}\right)$ and $K\left(f_{2}\right)$ are equal,

- the maps $f_{1}$ and $f_{2}$ are equal in a neighborhood of $K\left(f_{1}\right)$.

4.1. Hybrid class. We say that two polynomial like maps $f$ and $g$ are hybrid conjugated if there exists a quasiconformal map $\phi$ defined in a neighborhood of the filled-in Julia set of $f$ and with values in a neighborhood of the filled-in Julia set of $g$ such that $\phi \circ f=g \circ \phi$ and $\bar{\partial} \phi=0$ in $K(f)$. Now it is a classic result in the complex dynamics the theorem of Douady and Hubbard ([DH]) which asserts that any polynomial like map is hybrid conjugated with a polynomial. Moreover if $K(f)$ is connected then this polynomial is unique up conjugations by affine maps.

The following easy modification of the result of Douady and Hubbard will be a useful tool in the study of the polynomial like contrapart of the concept of multimodal map of type $n$.

Proposition 4.1 (Straightening lemma). Let $f: U_{1} \rightarrow U_{n+1}$ be a polynomial like map, which has the form $f=f_{n} \circ \cdots \circ f_{1}$, where $f_{i}: U_{i} \rightarrow U_{i+1}$ are ramified coverings of degree $N_{i}$ and $U_{i}$ are simply connected. Assume that the critical values of $f$ are contained in $U_{1}$. Then $f$ is hybrid conjugated with a polynomial in the form $P_{n} \circ \cdots \circ P_{1}$, where $P_{i}$ is a polynomial of degree $N_{i}$.

Proof. First of all, we can assume, using the uniformization Riemann mapping, that $U_{i}=D_{0}\left(r_{i}\right)=\left\{x:|x|<r_{i}\right\}$, for $i>1$, and $U_{1} \subset U_{2} \subset U_{3} \cdots \subset U_{n+1}$. Assume that the diameter of $U_{n+1}$ is very big. Hence $f_{i}: U_{i} \rightarrow U_{i+1}$ are polynomial like maps. We will obtain quasiregular extensions $\tilde{f}_{i}$ of $f_{i}$ and $\tilde{f}$ of $f$ whose are compatible: $\tilde{f}=\tilde{f}_{n} \circ \cdots \circ \tilde{f}_{1}$. Choose $\epsilon$ small and define $A_{n+1}:=\tilde{A}_{n+1}:=\left\{z \in \mathbb{C}: r_{n+1}-\epsilon \leq\right.$ $\left.|z| \leq r_{n+1}\right\}$ and $h_{n+1}=i d$. Suppose that we had defined $h_{i}: A_{i} \rightarrow \tilde{A}_{i}$, where $A_{i}$ is a very fine ring such that the external boundary of $A_{i}$ is exactly the boundary of $U_{i}$, $\tilde{A}_{i}$ is the pre-image of $A_{n+1}$ by $x^{N_{n} N_{n-1} \ldots N_{i}}$ and $h_{i}$ is an analytic homeomorphism. 


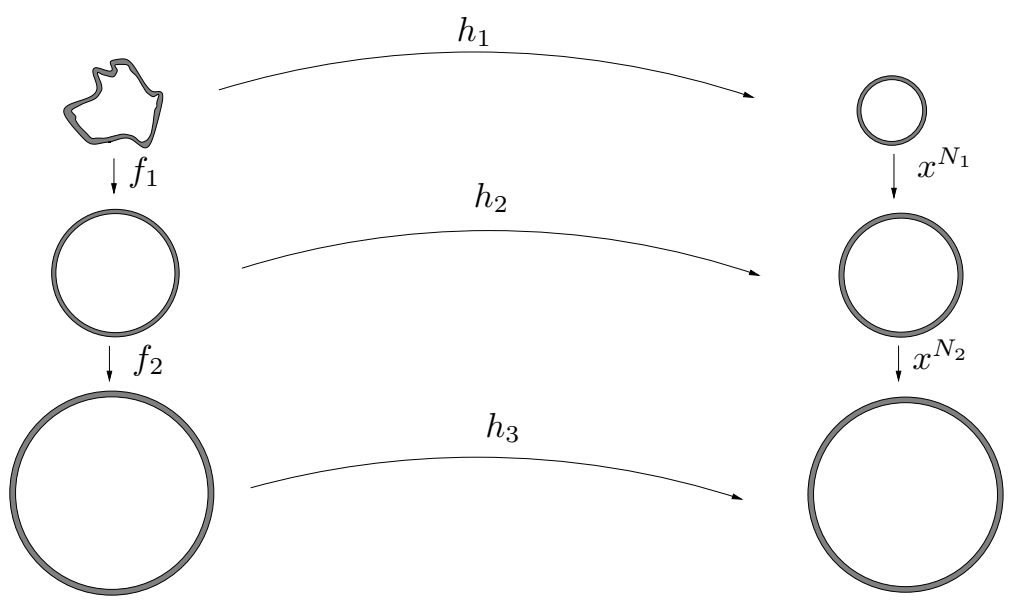

FiguRE 2

Define $A_{i-1}=f_{i-1}^{-1}\left(A_{i}\right)$ and $h_{i-1}$ as an analytic homeomorphism such that the following diagram commute

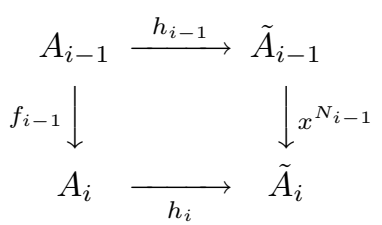

Let $H$ be a quasiconformal map which glues the maps $h_{i}$, extending the map to identity outside $U_{n+1}$. Now, we are able to define the quasiregular extensions

$$
\begin{gathered}
\tilde{f}_{i}(x)= \begin{cases}f_{i}(x) & x \in U_{i}, \\
H^{-1} \circ Q_{N_{i}} \circ H(x) & \text { in other case. }\end{cases} \\
\tilde{f}(x)= \begin{cases}f(x) & x \in U_{1}, \\
H^{-1} \circ Q_{N_{n} \ldots N_{1}} \circ H(x) & \text { in other case. }\end{cases}
\end{gathered}
$$

Here $Q_{n}(x):=x^{n}$. It is easy to see that these extensions are compatible. Make the pullback of the trivial Beltrami field outside $U_{n+1}$ by the quasiregular mapping $\tilde{f}$. We obtain an invariant Beltrami field $\mu$ for $\tilde{f}$ (defining the field trivial on $K(f)$ ). Define $\mu_{i}=\left(\tilde{f}_{n} \circ \cdots \circ \tilde{f}_{i}\right)_{*} \mu$. These Beltrami fields are trivial in a neighborhood of infinity. Let $L_{i}$ be the quasiconformal map so that $\frac{\partial L_{i}}{\bar{\partial} L_{i}}=\mu_{i}, L_{i}(0)=0, L_{i}(\infty)=\infty$, $L_{i}^{\prime}(\infty)=1$. Define $L_{n+1}=L_{1}$. Then $P_{i}=L_{i+1} \circ \tilde{f}_{i} \circ L_{i}^{-1}$ are polynomials. Moreover $P_{n} \circ P_{n-1} \cdots \circ P_{1}=L_{1}^{-1} \circ \tilde{f} \circ L_{1}$ is hybrid conjugated to $f$.

Now we are going to define the polynomial like analogous to the concept of multimodal map of type $n$ :

Definition 4.1. We say that $f: U \rightarrow V$ is a polynomial like map of type $\mathbf{n}$ if there exist simply connected domains $U=U_{1}, \ldots, U_{n}, U_{n+1}=V$ and holomorphic maps $f_{i}: U_{i} \rightarrow U_{i+1}, 1 \leq i \leq n$ satisfying

- $f_{i}: U_{i} \rightarrow U_{i+1}$ is a ramified covering map of degree two,

- $f=f_{n} \circ \cdots \circ f_{1}$. 
By the straightening lemma, any polynomial like map of type $n$ is hybrid conjugated with a polynomial in the form $P_{a_{n}} \circ \cdots \circ P_{a_{1}}$, where $P_{a}(x):=x^{2}+a$.

Remark 4.1. Note that we can assume, by the Riemann mapping lemma, that $U_{2}, \ldots, U_{n-1}$ are equal to $\mathbb{D}$.

We say that a polynomial like map $f: U_{0} \rightarrow U_{n}$ of type $n$ is real if there exists a decomposition $\left(f_{1}, \ldots, f_{n}\right), f_{i}: U_{i} \rightarrow U_{i+1}$, satisfying:

- The domains $U_{i}$ are symmetric which respect to the real axis and $I \subset U_{i}$,

- The maps $f_{i}$ preserves the interval $I$. Furthermore $f_{i}: I \rightarrow I$ is an unimodal map such that $\left(f_{1}, \ldots, f_{n}\right)$ is a decomposition for the multimodal map of type $n f: I \rightarrow I$.

4.2. External class. Let $f: U \rightarrow V$ be a polynomial like map of degree $d$ and connected filled-in Julia set. Consider $\phi: \overline{\mathbb{C}}-K(f) \rightarrow \overline{\mathbb{C}}-\overline{\mathbb{D}}$ be the Riemann mapping such that $\phi(\infty)=\infty$. Then the map $g=\phi \circ f \circ \phi_{-1}: \phi(U-K(f)) \rightarrow$ $\phi(V-K(f))$ is defined in an open set $A-\overline{\mathbb{D}}$, where $A$ is a neighborhood of $\partial \mathbb{D}$. We can invert $g$ along $\partial \mathbb{D}$ to obtain a holomorphic map $g: \tilde{U} \rightarrow \tilde{V}$ defined in a neighborhood of $S^{1}$. Here $\tilde{U}$ is the union of $\overline{\phi(U-K(f))}$ with its inversion along $S^{1}$. It is easy to see that $g$ is an expanding map of degree $d$. The map $g$ is called the external map of $f$. Note that $g$ is defined up to affine conjugacies. Indeed, the external map can be defined when $K(f)$ is not connected, but this will not be used here.

Proposition 4.2. Let $A$ and $B$ be neighborhoods of $S^{1}$ and $h: A-\overline{\mathbb{D}} \rightarrow B-\overline{\mathbb{D}}$ be a homeomorphism which commutes with $x^{d}$. Then $h$ has a continuous extension to $A-\mathbb{D}$ such that $h(z)=\alpha z$ in $S^{1}$, with $\alpha^{d-1}=1$.

Proof. Let

$$
A_{R}=\{z \in \mathbb{C}: 1<|z|<R\}
$$

and

$$
B_{R}=\{z \in \mathbb{C}: z=x+y \cdot i, \text { with } x, y \in \mathbb{R}, 0<|z|<\ln R\}
$$

Denote $Q(z)=z^{d}$ and define $\tilde{Q}: B_{R} \rightarrow B_{R^{d}}$ by $\tilde{Q}(z)=d \cdot z$ and $\mu(z)=e^{2 \pi z}$. The diagram

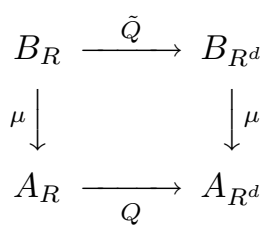

commutes. Fix an arbitrary $R>1$ and assume, without loss of generality, that the domain of $h$ is $A_{r}$, for some $r<R$ and $B-\overline{\mathbb{D}}$ is contained in $A_{R}$. Consider the fundamental annulus $A=Q^{-2}\left(A_{R^{d}}-A_{R}\right)$ and a compact set $\tilde{A} \subset B_{R}$ such that $\mu(\tilde{A})=A$. Then for any point $\tilde{z} \in \mu^{-1}\left(Q^{-2}\left(A_{R^{d}}\right)\right)$, there exist $j, i \in \mathbb{N}$ such that $\tilde{T}^{i}(\tilde{z})+j \in \tilde{A}$.

Select a homeomorphism $\tilde{h}$ such that $\mu \circ \tilde{h}=h \circ \mu$. The transformation $\tilde{h}$ is defined in the open set $\tilde{A} \cap A_{R}$ and with values in $\tilde{B} \cap A_{R}$, where $\tilde{A}=\mu^{-1}(A-\overline{\mathbb{D}})$ and $\tilde{B}=\mu^{-1}(B-\overline{\mathbb{D}})$. Since $h \circ T=T \circ h$, one gets

$$
\tilde{h} \circ \tilde{T}=\tilde{T} \circ \tilde{h}+k
$$


For some $k \in \mathbb{N}$ and for points in $A_{r^{1 / d}}$. Since

$$
(\tilde{h}+j) \circ \tilde{T}=\tilde{T} \circ(\tilde{h}+j)+k+j \cdot(d-1)
$$

we can assume, replacing $\tilde{h}$ by an appropriate translation of $\tilde{h}$ by an integer, that $0 \leq k<d-1$. Apply the equation 11 recursively to obtain

$$
\tilde{h} \circ \tilde{T}^{-i}=\tilde{T}^{-i} \circ \tilde{h}-k \cdot d^{-i} \cdot \frac{d^{i}-1}{d-1},
$$

for $j>0$ and points in $A_{r^{1 / d}}$. Let

$$
D=\sup _{z \in \tilde{A}} \operatorname{dist}_{B_{R}}\left(h(z), z-\frac{k}{d-1}\right)
$$

Then for any $\tilde{x} \in \mu^{-1}\left(Q^{-2}\left(A_{R^{d}}\right)\right.$, select $i, j$ such that $x=\tilde{T}^{-i}(z)-j$, with $z \in \tilde{A}$. If $\tilde{x} \in \tilde{A}$ then

$$
\begin{gathered}
\operatorname{dist}_{B_{R}}\left(\tilde{h}(\tilde{x}), \tilde{x}-\frac{k}{d-1}\right) \\
=\operatorname{dist}_{B_{R}}\left(\tilde{h}\left(\tilde{T}^{-i}(z)-j\right), \tilde{T}^{-i}(z)-j-\frac{k}{d-1}\right) \\
=\operatorname{dist}_{B_{R}}\left(d^{-i} \cdot \tilde{h}(z)-k \cdot d^{-i} \cdot \frac{d^{i}-1}{d-1}-j, d^{-i} \cdot z-j-\frac{k}{d-1}\right) \\
=\operatorname{dist}_{B_{R}}\left(d^{-i} \cdot\left(\tilde{h}(z)-k \cdot \frac{d^{i}-1}{d-1}-j \cdot d^{i}\right), d^{-i} \cdot\left(z-k \cdot \frac{d^{i}}{d-1}-j \cdot d^{i}\right)\right) \\
\leq \operatorname{dist}_{B_{R}}\left(\tilde{h}(z)+\frac{k}{d-1}-k \cdot \frac{d^{i}}{d-1}-j \cdot d^{i}, z-k \cdot \frac{d^{i}}{d-1}-j \cdot d^{i}\right) \\
=\operatorname{dist}_{B_{R}}\left(\tilde{h}(z), z-\frac{k}{d-1}\right) \leq D,
\end{gathered}
$$

The above proof is a variation of the Douady and Hubbard's proof $([\mathrm{DH}])$ when $k=0$. So $\operatorname{dist}_{A_{R}}(h(x), \alpha \cdot x) \leq D$, for $\mu(\tilde{x})=x$, because $\mu: B_{R} \rightarrow A_{R}$ is a local isometry. Hence $\alpha \cdot x-h(x) \rightarrow 0$ in the Euclidean topology, when $x \rightarrow S^{1}$, since $\rho_{A_{R}} \geq C \frac{1}{\operatorname{dist}\left(x, S^{1}\right)}$ near to $S^{1}$.

The above statement was proved by M. Lyubich([L1]) when $d=2$. We will denote $[h]=\alpha$. Notice $\left[h_{1} \circ h_{2}\right]=\left[h_{1}\right] \cdot\left[h_{2}\right]$, for two homeomorphisms $h_{i}$ which commutes to $x^{d}$.

Definition 4.2. Let $f: U \rightarrow V$ and $g: \tilde{U} \rightarrow \tilde{V}$ be polynomial-like maps. Let $h_{1}: U-K(f) \rightarrow \tilde{U}-K(g)$ and $h_{2}: U-K(f) \rightarrow \tilde{U}-K(g)$ be two homeomorphisms such that $g=h_{i} \circ f \circ h_{i}^{-1}$. Then $h_{1} \circ h_{2}^{-1}$ is an automorphism of $f$ in $U-K(f)$. Choose a homeomorphisms $\phi: U-K(f) \rightarrow A(1, r)$ such that $\phi \circ f=T \circ \phi$, with $T(x)=x^{d}$ and $d=\operatorname{deg} f$. Then $\phi \circ h_{1} \circ h_{2}^{-1} \circ \phi^{-1}$ is an automorphism of T. Define

$$
\left[f, g ; h_{1}, h_{2}\right]=\left[\phi \circ \psi \circ \phi^{-1}\right]
$$

Observe that $\left[f, g ; h_{1}, h_{2}\right]$ is well defined, since if $\phi_{1}$ and $\phi_{2}$ are two conjugacies between $f$ and $T$ then

$$
\left[\phi_{1} \circ \psi \circ \phi_{1}^{-1}\right]=\left[\phi_{1} \circ \phi_{2}^{-1}\right] \cdot\left[\phi_{2} \circ \psi \circ \phi_{2}^{-1}\right] \cdot\left[\left(\phi_{1} \circ \phi_{2}^{-1}\right)^{-1}\right]=\left[\phi_{2} \circ \psi \circ \phi_{2}^{-1}\right]
$$

The number $\left[f, g ; h_{1}, h_{2}\right]$ was introduced by Douady and Hubbard([DH]) to study when it is possible to glue conjugacies: 
Corollary 4.1 (Gluing conjugacies:[DH]). Let $f$ and $g$ be polynomial like maps, let $h_{1}: V_{f} \rightarrow V_{g}$ and $h_{2}: V_{f}-K(f) \rightarrow V_{g}-K(g)$ be conjugacies. If $\left[f, g ; h_{1}, h_{2}\right]=1$ then there exists a conjugacy $h: V_{f} \rightarrow V_{g}$ such that

- The map $h$ coincides with $h_{1}$ in $K(f)$.

- The map $h$ coincides with $h_{2}$ in $V_{f}-K(f)$.

Corollary 4.2. Let $f: U \rightarrow V$ and $g: \tilde{U} \rightarrow \tilde{V}$ be polynomial like maps with the same hybrid and external class. If there is an external equivalence $h_{1}$ and a hybrid equivalence $h_{2}$ such that $\left[f, g, h_{1}, h_{2}\right]=1$, then $f$ and $g$ are affine conjugated.

4.3. Compact sets. In a neighborhood of the locus of connectivity of polynomials of type $n$, select a holomorphic moving fundamental annulus $A_{p}$. This means that for each $p \in \mathcal{C}_{n}$ there exist a neighborhood $\Lambda$ of $p$ and a map $\psi: \Lambda \times A_{p} \rightarrow \mathbb{C}$ such that

- For each $\tilde{p} \in \Lambda, \psi\left(\tilde{p}, A_{p}\right)=A_{\tilde{p}}$.

- For each point $z$ in the annulus $A_{p}, \psi(\cdot, z)$ is a holomorphic function.

- If $U_{p}$ and $V_{p}$ are respectively bounded simply connected domains whose boundaries are the internal and external boundaries of the annulus $\psi\left(p, A_{p}\right)$, then $p: U_{p} \rightarrow V_{p}$ is a polynomial-like map.

To find such $\phi$, let $C$ be a large circle centered in zero which contains all the Julia sets for polynomials of type $n$ contained in the locus of connectivity. Then, for $p$ near to $\mathcal{C}, p^{-1}(C)$ is a Jordan curve contained in the disc whose boundary is $C$. We obtain a polynomial-like representation for $p$. Furthermore the set $C \cup p^{-1}(C)$ moves holomorphically in a neighborhood of $p$. Let $\psi$ be this holomorphic motion. This holomorphic motion can be extended for all points in $\mathbb{C}$. Then we define $A_{\tilde{p}}$ as the annulus delimited by $C$ and $\tilde{p}^{-1}(C)$. We will fix this holomorphic moving annulus on the polynomials of type $n$ in the rest of this paper.

Definition 4.3. We say $f: U \rightarrow V$, a polynomial like map of type $n$ with connected Julia set, belongs to $\mathcal{P}_{n}(C)$ if there are a $C$-quasiconformal map $\phi: \mathbb{C} \rightarrow \mathbb{C}$ and $p \in \mathcal{C}_{n}$ such that

$$
\begin{aligned}
& \text { - } \phi\left(V_{p}-U_{p}\right)=V-U \text {, } \\
& \text { - } \phi \circ p=f \circ \phi \text { in } U_{p} \text {. }
\end{aligned}
$$

Proposition 4.3. The set $\mathcal{P}_{n}(C)$ is compact up to affine conjugacies.

Proof. Let $f_{i}$ be a sequence in $\mathcal{P}_{n}(C)$. Replacing $f_{i}$ by a polynomial like map which is affine conjugated to it, we can assume $\operatorname{diam} K\left(f_{i}\right)=1$. Consider $C$ quasiconformal maps $\phi_{i}$ and polynomial maps $p_{i}$ as in definition 4.3. Since $\mathcal{C}_{n}$ is compact, select a subsequence, if necessary, such that $p_{i} \rightarrow p \in \mathcal{C}_{n}$. Since the Julia set of $p \in \mathcal{C}_{n}$ has the diameter away of infinity and zero, and $\phi_{i}\left(K\left(f_{i}\right)\right)=K\left(p_{i}\right)$, selecting a subsequence we can assume that $\phi_{i}$ converges to a $C$-quasiconformal map. It is not difficult to see that $\phi \circ p \circ \phi^{-1}$ is an analytic map in $\phi\left(V_{p}-U_{p}\right)$.

For a set $K \subset \mathbb{C}$, denote $\delta$ - $K:=\{z \in \mathbb{C}: \operatorname{dist}(z, K)<\delta \operatorname{diam} K\}$.

Corollary 4.3. For any $\delta>0$ there exists $n=n(C, \delta)$ so that if $f: U \rightarrow V$ belongs to $\mathcal{P}_{n}(C)$ then $f^{-n}(V) \subset \delta-K(f)$.

Proof. Easy. 
Lemma 4.1 ([McM2]). Let $f: U \rightarrow V$ be a polynomial like map with connected filled-in Julia set and $\epsilon-K(f) \subset V$. Then the germ of $f$ has a representation $f: \tilde{U} \rightarrow \tilde{V}$ such that:

- The boundaries of $\tilde{U}$ and $\tilde{V}$ are $C(\epsilon)$-quasicircles,

- $\operatorname{diam} \tilde{V} \leq \tilde{C}(\epsilon) \operatorname{diam} K(f)$,

- $\bmod (\tilde{V}-\tilde{U}) \geq m(\epsilon)$.

When $p$ is a polynomial of type $n$ with connected Julia set, we can select a polynomial like restriction $p: \tilde{U}_{0} \rightarrow \tilde{U}_{n}$ with the above properties in the following way: Let $\phi: \mathbb{C}-K(f) \rightarrow \mathbb{C}-\mathbb{D}$ be the Riemann map such that $\phi(\infty)=\infty$. We have

$$
\phi \circ p(x)=(\phi(x))^{d}
$$

for $x \in \mathbb{C}-K(p)$ and $d=\operatorname{deg} p$. Let $\mathbb{D}_{r}=\{z \in \mathbb{C}:|z| \geq r\}$. Define

$$
U_{0}=\phi^{-1}\left(\mathbb{D}_{\exp \frac{m}{d-1}}-\overline{\mathbb{D}}\right) \cup K(p) \text { and } U_{n}=\phi^{-1}\left(\mathbb{D}_{\exp \frac{d m}{d-1}}-\overline{\mathbb{D}}\right) \cup K(p)
$$

Then $\bmod \left(U_{n+1}-U_{0}\right)=m$. It is easy to prove that $\partial \tilde{U}_{0}$ and $\partial \tilde{U}_{n}$ are $C(m)$ quasicircles. To prove that $\operatorname{diam} \tilde{U}_{n} \leq \tilde{C}(m) \operatorname{diam} K(p)$, recall that the diameter of $K(p)$ is bounded above and below, by lemma 3.1. So it is sufficient to prove that $\operatorname{diam} U_{n+1} \leq C(m)$. Indeed, consider the Green function $G(x)=\log |\phi(x)|$. Since $\mathcal{C}$ is compact, for any $\epsilon>0$ there exists $R_{\epsilon}$ such that for $|z| \geq R_{\epsilon}$ and $p \in \mathcal{C}$ one have

$$
\frac{1}{d} \log (1-\epsilon)+\log |z| \leq G(x)
$$

( e.g., see the proof of Theorem 2.1 in [FS]), which clearly implies diam $U_{n+1} \leq$ $C(m)$, since $G\left(\partial U_{n+1}\right)=d m /(d-1)$. The advantage of this polynomial like restriction is that the annulus $U_{n+1}-K(f)$ and $U_{0}-K(f)$ are invariant by the external automorphisms

$$
\phi^{-1} \circ R_{\alpha} \circ \phi: \mathbb{C}-K(f) \rightarrow \mathbb{C}-K(f)
$$

where $R_{\alpha}(x)=\alpha x$ and $\alpha^{d-1}=1$.

Denote by $\mathcal{P}_{n}(C, \tilde{C}, m)$ the set of polynomials like maps of type $n$ which admits a decomposition $f=f_{n} \circ \cdots \circ f_{1}: U_{1} \rightarrow U_{n+1}, f_{i}: U_{i} \rightarrow U_{i+1}$, such that

- The filled-in Julia set $K(f)$ is connected;

- The boundaries of $U_{i}$ are either $C$-quasicircles, for $i=1, n+1$, or the unit disc, otherwise;

- $\operatorname{diam} U_{n+1} \leq \tilde{C} \operatorname{diam} K(f)$;

- $\bmod \left(U_{n+1}-U_{1}\right) \geq m$;

- The critical point of $f_{i}$ is 0 .

Proposition 4.4. Let $f: U_{1} \rightarrow U_{n}$ and $\tilde{f}: \tilde{U}_{1} \rightarrow \tilde{U}_{n}$ be polynomial like maps in of type $n$ which belongs $\mathcal{P}_{n}(C, \tilde{C}, m)$ which are conjugated by a one-to-one continuous map $h_{0}$ in a neighborhood of their filled-in Julia sets. Then there exists a one-to-one continuous map $h_{1}: \mathbb{C} \rightarrow \mathbb{C}$ between $f$ and $\tilde{f}$ with the following properties:

- The map $h_{1}$ is a conjugacy: $\tilde{f} \circ h_{1}=h_{1} \circ f$ in $U_{1}$

- $h_{1}\left(U_{n}-U_{1}\right)=\tilde{U}_{n}-\tilde{U}_{1}$.

- $h_{1}=h_{0}$ in $K(f)$.

- $h_{1}$ is $C(C, \tilde{C}, m)$-quasiconformal in $\mathbb{C}-K(f)$.

In particular, if $h_{0}$ is a hybrid conjugacy then $h_{1}$ is a hybrid conjugacy. 
Proof. Assume that $\operatorname{diam} K(f)=\operatorname{diam} K(\tilde{f})=1$. Note that, since the boundary of $U_{i}$ is a quasicircle, the map $f_{i}$ has a quasiregular extension in a neighborhood of $\overline{U_{i}}$. Indeed, let $\alpha_{i}: \mathbb{C} \rightarrow \mathbb{C}$ be a $C\left(C_{1}\right)$-quasiconformal map which is conformal in $\mathbb{D}$ and maps $\mathbb{D}$ in $U_{i}$. Then $\alpha_{i+1}^{-1} \circ f_{i} \circ \alpha_{i}: \mathbb{D} \rightarrow \mathbb{D}$ extends to a rational map $g_{i}$ which is a expansive map of degree 2 in $S^{1}$. Hence $\alpha_{i+1} \circ f_{i} \circ \alpha_{i}^{-1}: \mathbb{C} \rightarrow \mathbb{C}$ is a regular map of degree two in a neighborhood of $\bar{U}_{i}$. The same can be done for $\tilde{f}_{i}$. Let $\phi_{n}: \mathbb{C} \rightarrow \mathbb{C}$ be a $C_{n}(C)$-quasiconformal map which maps $U_{n}$ in $\tilde{U}_{n}$. Since $f_{n}(0)$ and $\tilde{f}_{n}(0)$ are contained in the Julia sets of $f$ and $\tilde{f}$, these points are at a definitive Euclidean distance of $\partial U_{n+1}$ and $\partial \tilde{U}_{n+1}$. Thus, by lemma 8.5 , we can assume that $\phi_{n}\left(f_{n}(0)\right)=\tilde{f}_{n}(0)$.

We will prove, by induction, that there are $C_{i}(C, m)$-quasiconformal maps $\phi_{i}: U_{i} \rightarrow$ $\tilde{U}_{i}$, such that

- $\phi_{i+1} \circ f_{i}=\tilde{f}_{i} \circ \phi_{i}^{-1}$ in $\partial U_{i}$.

- $\phi_{i}\left(f_{i}(0)\right)=\tilde{f}_{i}(0)$.

Let $\phi_{i-1}: U_{i-1} \rightarrow \tilde{U}_{i-1}$ be a lift of $\phi_{i}$ (in other words: $\phi_{i} \circ f_{i-1}=\tilde{f}_{i-1} \circ \phi_{i-1}^{-1}$ ), which has the same quasiconformality than $\phi_{i}$. Because the critical values of $f$ does not intercept $U_{n+1}-U_{1}$, and the modulus of this annulus is bounded below, $f_{i-1}(0)$ is at a bounded distance of 0 in the hyperbolic metric on $U_{i-1}$. Since the same can be said about $\tilde{f}_{i-1}(0)$, by lemma 8.5 , if necessary modify $\phi_{i-1}$ such that $\phi_{i-1}\left(f_{i-1}(0)\right)=$ $\tilde{f}_{n-1}(0)$, and additionally the new $\phi_{i-1}$ is $C_{i-1}(C, m)$-quasiconformal. In particular $\phi_{n} \circ f=\tilde{f} \circ \phi_{0}$ in $\partial U_{0}$. By lemma 5.3.1 in [GS], we can find a $C(C, m, M)$ quasiconformal map $H: \mathbb{C} \rightarrow \mathbb{C}$ such that (1) $H$ is equal to $\phi_{n+1}$ outside $U_{n+1}(2)$ $H$ is equal to $\phi_{1}$ in $\overline{U_{1}}$. Hence $H$ is a quasiconformal map which maps $\overline{U_{n+1}-U_{1}}$ in $\overline{\tilde{U}_{n+1}-\tilde{U}_{1}}$ and conjugates $f$ and $\tilde{f}$ in the boundary of this fundamental annulus. Now, with the usual pullback argument, construct a $C(C, m, M)$-quasiconformal conjugacy $H$ between $f$ and $\tilde{f}$ in $U_{n+1}-K(f)$ in $\tilde{U}_{n+1}-K(\tilde{f})$ such that $f\left(U_{n+1}-\right.$ $\left.U_{1}\right)=\tilde{U}_{n+1}-\tilde{U}_{1}$. For the last step, to obtain a conjugacy which extends to $K(f)$, the result follows of the particular case when $\tilde{f}$ is a polynomial and the annulus $\tilde{U}_{n+1}-\tilde{U}_{1}$ is invariant by the external automorphisms of $\tilde{f}$. Select an external automorphism $R: \mathbb{C}-K(\tilde{f}) \rightarrow \mathbb{C}-K(\tilde{f})$ so that $[h, R \circ H ; f, \tilde{f}]=1$. Thus the conjugacy $h_{0}$ in $K(f)$ glues with the external conjugacy $R \circ H$ and the new map $h_{1}$ has the same quasiconformality of $H$ outside $K(f)$.

\section{RenORMALIZATION}

5.1. Infinitely renormalizable polynomials. Here we will work with a more natural parameterization of polynomial of type $n$. We will consider the family of polynomials in the form $f=f_{a_{1}} \circ \cdots \circ f_{a_{n}}$, where $f_{a}(x)=-2 a x^{2}+2 a-1, a \in \mathbb{C}$. Note that $\alpha f(1 / \alpha)$ belongs to $\mathrm{Pol}_{n}$, with

$$
\alpha^{2^{n}-1}=-2^{2^{n}-1} a_{1} a_{2}^{2} a_{3}^{4} \ldots a_{n}^{2^{n-1}} .
$$

For each $f$ in this family there is at most $2^{n}-1$ polynomials in $\mathrm{Pol}_{n}$ affine conjugated to it. Furthermore, if $a_{i} \in \mathbb{R}$ then there is exactly a real map in Pol $_{n}$ which is affine conjugated to $f$. Because the results of section 2.1, there is, for each combinatorial type $\sigma=\left(\sigma_{1}, \sigma_{2}, \ldots\right)$, at least one infinitely renormalizable multimodal map of type $n$ in this family which has type $\sigma$. Denote the set of infinitely renormalizable real polynomials of type $n$ by $\mathrm{Pol}_{n}^{\infty}$ and the subset of $\operatorname{Pol}_{n}^{\infty}$ with $C$-bounded combinatorics by $\operatorname{Pol}_{n}^{\infty}(C)$. Denote by $F$ the extended 
map associated to a decomposition in quadratic polynomials $\left(f_{a_{1}}, \ldots, f_{a_{n}}\right)$. The bounded geometry of the postcritical set of $f \in \operatorname{Pol}_{n}^{\infty}(C)$ is consequence of results of Blokh and Lyubich $([\mathrm{BL}])$ (see also J. Hu's thesis[H1] and [H2] ). The following result can be proved as in [Sm1](for notation, see introduction):

Proposition 5.1 (Bounded geometry). Let $q, r, s$ be arbitrary critical points of $F$ so that $Q_{-i}^{k+1}$ and $R_{-j}^{k+1}$ are contained in $S_{-\ell}^{k}$. The following quantities are $C_{1}(C)$ commensurable:

- The lengths of $Q_{-i}^{k+1}, R_{-j}^{k+1}$ and $S_{-\ell}^{k}$,

- The distance between $Q_{-i}^{k+1}$ and $\partial S_{-\ell}^{k}$,

- The distance between $Q_{-i}^{k+1}$ and $R_{-j}^{k+1}$, if these intervals do not touch.

Denote by $\mathcal{P}_{n}^{\infty}\left(C_{1}, C_{2}\right)$ the set of maps in $\mathcal{P}_{n}\left(C_{2}\right)$ which are hybrid conjugated with polynomials in $\operatorname{Pol}_{n}^{\infty}\left(C_{1}\right)$. The main technical result in renormalization theory is

Proposition 5.2 (Complex Bounds [Sm1]). Let $f$ be a map in $\operatorname{Pol}_{n}^{\infty}\left(C_{1}\right)$. Then there exist $k_{0}\left(C_{1}\right)$ and $C_{2}\left(C_{1}\right)$ so that any renormalization $R^{k}(f), k \geq k_{0}$, has a polynomial-like extension $R^{k}(f): U \rightarrow V$ in $\mathcal{P}_{n}^{\infty}\left(C_{1}, C_{2}\right)$. Furthermore, the renormalization is unbranched: $P(f) \cap V=P(f) \cap K\left(R^{k}(f)\right)$.

Proof. Here we have a very nice situation: the map $\mathrm{f}$ is a polynomial, it has negative Schwartzian derivative and moreover satisfies properties analogous to the standard conditions (see [Sm1]). It is easy verify in the proof of the complex bounds for analytic multimodal maps [Sm1] that $k_{0}$ and $C_{2}$ (for this use lemmas 4.1 and 4.4) can be select independent of $f \in \operatorname{Pol}_{n}^{\infty}\left(C_{1}\right)$.

Indeed, we proved the complex bounds for any analytic map $f$ which is infinitely renormalizable with bounded combinatorics. But, in this case, $k_{0}$ depends on $f$. Anyway, now we can assume in the Main Theorem that $f$ and $g$ (and all renormalizations) are contained in $\mathcal{P}_{n}^{\infty}\left(C_{1}, C_{2}\right)$.

$\mathrm{J} . \mathrm{Hu}([\mathrm{H} 1])$ stated a complex bounds result for bimodal maps in the Epstein class and bounded combinatorics, but the outline of the proof seems to be incomplete. Recently W. Shen([Sh2]) claimed complex bounds for any infinitely renormalizable analytic map.

Proposition 5.3. The following statements holds:

- Let $f_{i}$ be maps in $\operatorname{Pol}_{n}^{\infty}(C)$ with combinatorics $\sigma_{n}$. If $\sigma_{n}$ converges to $\sigma$ then any limit $f_{\infty}$ of a subsequence of $f_{i}$ has combinatorics $\sigma$.

- The postcritical set moves continuously in $\mathrm{Pol}_{n}^{\infty}(C)$.

Proof. Let $P_{i}^{k}$ be the restrictive interval associated to the $k$-th renormalization of $f_{i}, 0 \in P_{i}^{k}$. Since the period of $P_{i}^{k}$ is bounded by $p_{0}(k, C)$, the length of $P_{i}^{k}$ can not be small, otherwise $f_{i}$ will contain a periodic point which attracts zero, which is impossible. So we can assume that $P_{i}^{k}$ converges to a periodic interval $P_{\infty}^{k}$ for $f_{\infty}$, which proves that $f_{\infty}$ belongs to $\operatorname{Pol}_{n}^{\infty}(C)$. In particular, all periodic points of $f_{\infty}$ in $I$ are repelling (because non repelling periodic points attracts a critical point), so the periodic point in the boundary of $P_{i}^{k}$ converges to a periodic point in $\partial P_{\infty}^{k}$. Thus $P_{i}^{k}$ is the unique restrictive interval associated to the $k$-th renormalization of $f_{\infty}$ and the $k$-th restrictive interval moves continuously in $\operatorname{Pol}_{n}^{\infty}(C)$ and so do the postcritical set. 
5.2. Renormalization for polynomial like maps. Let $f: U \rightarrow V$ be a polynomial like map. A pre renormalization of $f$ is a polynomial like map $g: \tilde{U} \rightarrow \tilde{V}$ such that

(1) $\tilde{U} \subset U$,

(2) $g=f^{i}$ for some $i>0$,

(3) The filled-in Julia set $K(g)$ is connected.

Note that a pre-renormalization of a polynomial-like map of type $n$ is a polynomial like map of type $k$, for some $k \geq 0$. This is a consequence of the following observation: if $g_{1}$ and $g_{2}$ are holomorphic maps such that $g_{2} \circ g_{1}: U \rightarrow V$ is a proper map, then $g_{1}: U \rightarrow g_{1}(U)$ and $g_{2}: g_{1}(U) \rightarrow V$ are proper maps.

Lemma 5.1. Let $f: U \rightarrow V$ be a polynomial like map in $\mathcal{P}_{n}(C)$ with a pre renormalization $g=f^{m}: \tilde{U} \rightarrow \tilde{V}$ such that $c \in K(g)$, where $c$ is the critical point mapped to zero by conjugacies with a polynomial of type $n$. Then $\operatorname{diamK}(g) \geq$ $C_{1}(C, m) \operatorname{diamK}(f)$

Proof. Follows of lemma 3.3.

Lemma 5.2. Let $g_{1}=f^{n}: U_{1} \rightarrow V_{1}$ and $g_{2}=f^{n}: U_{2} \rightarrow V_{2}$ be pre renormalizations of a polynomial like map $f$. Consider $K=K\left(g_{1}\right) \cap K\left(g_{2}\right)$. Then one of the following statements holds:

(1) $K=\phi$.

(2) $K=\{p\}$, where $p$ is a repelling periodic point of $f$.

(3) $K$ is the filled-in Julia set of a pre-renormalization $g: \tilde{U} \rightarrow \tilde{V}$ of $f$. Moreover $\operatorname{deg}(g) \leq \min \left\{\operatorname{deg}\left(g_{1}\right), \operatorname{deg}\left(g_{2}\right)\right\}$ and the equality holds iff $K(g)=$ $K\left(g_{1}\right)$ or $K(g)=K\left(g_{2}\right)$.

Proof. Follows of the connectedness principle by McMullen (pg. 90 in [McM1]) that $K=K\left(g_{1}\right) \cap K\left(g_{2}\right)$ is connected. Let $\tilde{U}$ be the connected component of $U_{1} \cap U_{2}$ which contains $K$. Then $g=f^{n}: \tilde{U} \rightarrow f^{n}(\tilde{U})$ is a polynomial like map, and moreover $K(g)=K$, since $K$ is totally invariant by $g$. Hence we obtain item 2 , if $\operatorname{deg} g=1$, or 3 , otherwise. The last statement of item 3 follows of lemma 5.11 in $[\mathrm{McM} 1]$.

Remark 5.1. A special case is when each critical point of the extended map F associated to $f$ can be accumulated by points in the closure of the postcritical orbit of c. In this case if $g_{i}=f^{k}: U_{i} \rightarrow V_{i}, i=1,2$ are two pre renormalizations with $\operatorname{deg} g_{i} \leq 2^{n}$ then or $K\left(g_{1}\right)=K\left(g_{2}\right)$ and $\operatorname{deg} g_{i}=2^{n}$ either $K\left(g_{1}\right) \cap K\left(g_{2}\right)$ is at most a repelling period point. In particular if $g$ is a pre-renormalization of $f$ whose domain contains $c$ and it has degree at most $2^{n}$ then $\operatorname{deg} g=2^{n}$ and any pre renormalization of $g=f^{k}: U \rightarrow V$, for fixed $k$, whose domain contains $c$ define the same germ of polynomial like map of type $n$. Then we can call this germ $g$ as the renormalization of $f$.

We do not know if there is a canonical way of define renormalization when $f$ do not satisfy the hypothesis in the previous remark. However, in the case of real polynomial like map $f: U \rightarrow V$ of type $n$ where $\partial P$ contains a repelling period point, we can use external rays which arrive in the boundary points of the restrictive interval $P$ to find a degenerate polynomial like extension to the renormalization. After modify the domain near to the boundary points of $\mathrm{P}$, we obtain: 
Proposition 5.4. Let $f: U \rightarrow V$ be a real polynomial like map of type $n$ which is renormalizable in the sense of section 1.1. Let $P$ be the restrictive interval associated with the renormalization $R f$. Assume that $\partial P$ contains a repelling period point. Let $k$ be minimal such that $f^{k}(P) \subset P$. Then there exists a pre renormalization $\tilde{g}: \tilde{U} \rightarrow \tilde{V}$ of degree $2^{n}$ such that $K(\tilde{g}) \cap \mathbb{R}=P$.

Remark 5.2. If $g_{1}$ and $g_{2}$ are two pre renormalizations of degree $2^{n}$ such that the restrictive interval $P$ is contained in $K\left(g_{i}\right)$. Then $K\left(g_{1}\right) \cap K\left(g_{2}\right)$ is the filled in Julia set of a pre-renormalization $g$. Since $K\left(g_{1}\right) \cap K\left(g_{2}\right)$ contains $P, \operatorname{deg}(g)=2^{n}$. By lemma 5.2 $K(g)=K\left(g_{1}\right)=K\left(g_{2}\right)$. Thus $g_{i}$ define the same polynomial like germ. This germ will be called the complex renormalization of $f$. We say that a polynomial like map of type $n$ is renormalizable if it is hybrid conjugated with a renormalizable real polynomial like map of type $n$. Denote by $\mathcal{P}_{n}^{\infty}\left(C_{1}, C_{2}\right)$ the subset of maps in $\mathcal{P}_{n}\left(C_{2}\right)$ which are hybrid conjugated with a real infinitely renormalizable polynomial of type $n$ with combinatorics bounded by $C_{1}$.

Let $K$ be a closed set in $\mathbb{C}$. We say that $K$ has $C$-bounded geometry if $1 / C<\sup _{A \in \mathcal{A}} \bmod A<C$ where $\mathcal{A}$ is the set of annulus $A \subset \mathbb{C}-K$ such that the both components of $\mathbb{C}-A$ contain points in $K$. Recall that if $R^{j} f=f^{m(j)}: U \rightarrow V$ is a renormalization of $f$, we call the sets

$$
K\left(R^{j} f\right), F\left(K\left(R^{j} f\right)\right), \ldots, F^{m(j)-1}\left(K\left(R^{j} f\right)\right)
$$

the small Julia sets of $f$. The following result will be used a lot of times:

Proposition 5.5. For $f \in \mathcal{P}_{n}^{\infty}\left(C_{1}, C_{2}\right)$, the followings holds:

(1) $K(f)$ has empty interior.

(2) For almost every point $x$ in the Julia set, $f^{n}(x) \rightarrow P(f)$.

(3) The small Julia sets touch at most in an unique point.

(4) The postcritical set $P(f)$ and $K(f)$ moves continuously in $\mathcal{P}_{n}^{\infty}\left(C_{1}, C_{2}\right)$.

(5) There exists a constant $C\left(C_{1}, C_{2}\right)$ such that the postcritical set $P(f)$ has C-bounded geometry.

(6) The $k$-th renormalization, for $k \geq k_{0}(C)$, has a polynomial-like extension $R^{k}(f): U^{k} \rightarrow V^{k}$ which belongs to $\mathcal{P}_{n}^{\infty}\left(C, C_{2}\right)$. Here $C=C\left(C_{1}, C_{2}\right)$.

(7) There exists $j\left(C_{1}, C_{2}\right)$ so that

$$
\frac{\operatorname{diam} K\left(R^{k+j}(f)\right)}{\operatorname{diam} K\left(R^{k}(f)\right)} \leq \frac{1}{2}
$$

Proof. The proof of 1 is exactly as in the unimodal case (see [McM1]): we can assume that $f$ is a polynomial. Suppose, by contradiction that $K(f)$ has interior. Then, by the Sullivan's classification of periodic components, the interior of $K(f)$ contains an attractor or a Siegel disc: the first case is impossible because $P(f)$ is a Cantor set (and any attractor attracts a critical point) and the second one does not hold because the boundary of a Siegel disc must be contained in the postcritical set. The second statement is consequence of the ergodic or attract theorem([McM1]). Item 3 is consequence of remark 5.1. Item 4 and 5 follow of the same statements for polynomials (propositions 5.3 and 5.2). The last item is obvious for polynomials, since $\operatorname{diam} K\left(R^{k}(f)\right)$ is commensurable with the length of $P_{0}^{k}$, which goes exponentially fast to zero (proposition 5.1). Now the general case is easy. 
If $M$ is a hyperbolic Riemann surface, denote by $\|\cdot\|_{M}$ the hyperbolic metric in $T M$ and $\operatorname{dist}_{M}(\cdot, \cdot)$ the hyperbolic distance. We will denote by $\operatorname{dist}(\cdot, \cdot)$ the Euclidean distance.

Proposition 5.6. There exists a constant $D$ such that, for any $f: U \rightarrow V \in$ $\mathcal{P}_{n}^{\infty}\left(C_{1}, C_{2}\right)$,

for $z \in \overline{f^{-1}(V-U)}$.

$$
\operatorname{dist}_{V-P(f)}\left(z, f^{-1}(P(f))\right) \leq D
$$

Proof. It is easy to see that there is a bound for $\operatorname{diam}_{V-P(f)} \overline{f^{-1}(V-U)}$ which depends only on $C_{1}$ and $C_{2}$. So it suffice to prove that there is a point $z \in \overline{f^{-1}(V-U)}$ whose hyperbolic distance to $f^{-1}(P(f))$ is under control. Firstly, assume that $p: U \rightarrow V$ is a polynomial in $\operatorname{Pol}_{n}^{\infty}\left(C_{1}\right)$ and $V_{p}-\overline{U_{p}}$ is the holomorphically moving fundamental annulus $A_{p}$ selected in section 4.3. We will prove that there exists $\tilde{D}$ such that for each $p \in \operatorname{Pol}_{n}^{\infty}\left(C_{1}\right)$ there exist points $x_{p} \in V_{p}-\overline{U_{p}}, y_{p} \in p^{-1}(P(p))$ and a topological disc $B_{p}$ such that $x_{p}, y_{p} \in B_{p}$ and $\overline{B_{p}} \subset V_{p}-P(p)$ satisfying:

$$
\operatorname{dist}_{B_{p}}\left(x_{p}, y_{p}\right) \leq \tilde{D}
$$

Indeed, for each $p$ with combinatorics bounded by $C_{2}$, select a point $z_{0} \in \overline{f^{-1}\left(V_{p}-U_{p}\right)}$ and a topological disc $B$ which contains $z_{0}$ and a point $z_{1} \in p^{-2}\{0\}-P(p)$. Furthermore $\bar{B} \subset V_{p}-P(p)$. since $P(p)$ and $\partial V_{p}$ moves continuously with $p$, for $\tilde{p}$ close to $p$ we have $\bar{B} \subset V_{\tilde{p}}-P(\tilde{p})$. Furthermore there is a point $\tilde{z}_{1}$ of $\tilde{p}^{-2}(0)-P(\tilde{p})$ close to $z_{1}$. In particular, $\operatorname{dist}_{B}\left(z, \tilde{z}_{1}\right)$ is under control. Since $\operatorname{Pol}_{n}^{\infty}\left(C_{1}\right)$ is compact, the proof is finished for maps in $\operatorname{Pol}_{n}^{\infty}\left(C_{1}\right)$. In the general case, let $f: U \rightarrow V \in \mathcal{P}_{n}^{\infty}\left(C_{1}, C_{2}\right)$. Then there exists a $C_{2}$-quasiconformal conjugacy $\phi$ between $f$ and a polynomial $p: U_{p} \rightarrow V_{p} \in \operatorname{Pol}_{n}^{\infty}\left(C_{1}\right)$ such that $\phi\left(V_{p}-\bar{U}_{p}\right)=V-U$. But this implies

$$
\operatorname{dist}_{\phi\left(B_{p}\right)}\left(\phi\left(x_{p}\right), \phi\left(y_{p}\right)\right) \leq D_{1},
$$

where $D_{1}$ depends only on $C_{1}$ and $C_{2}$. Since that $\phi\left(x_{p}\right) \in V-\bar{U}$ and $\phi\left(y_{p}\right) \in$ $f^{-1}(P(f))$, this is enough to finish the proof, as in the polynomial case.

Let $f: U_{1} \rightarrow U_{n+1}$ be a polynomial like map of type $n$. For each decomposition $f_{i}: U_{i} \rightarrow U_{i+1}$, we can associate the extended map $F: \mathcal{U} \rightarrow \mathcal{V}$, where $\mathcal{U}=\left\{(x, i): x \in U_{i}, 1 \leq i \leq n\right\}$ and $\mathcal{V}=\left\{(x, i): x \in U_{i}, 2 \leq i \leq n+1\right\}$, defined by

$$
F(x, i)=\left(f_{i}(x), i+1 \bmod n\right)
$$

Thus $F$ is a ramified covering map between the Riemann surfaces $\mathcal{U}$ and $\mathcal{V}$. If $c_{i}$ is the critical point of $f_{i}$, define the postcritical set of $F$ by

$$
P(F)=\overline{\cup_{i} \cup_{j} F^{j}\left(c_{i}, i\right)}
$$

Assume now that $f \in \mathcal{P}_{n}^{\infty}\left(C_{1}, C_{2}\right)$. Then $P(F)$ is a Cantor set with bounded geometry. In particular $\mathcal{V}-P(F)$ is a $M\left(C_{1}, C_{2}\right)$-uniform domain (see Appendix). Clearly, by complex bounds, each small Julia set is the Julia set of a polynomial like map $g$ in $\mathcal{P}_{n}^{\infty}\left(C_{3}\left(C_{1}, C_{2}\right), C_{2}\right)$. Furthermore the fundamental annulus of $g$ does not intersect the postcritical set of $F$. For each small Julia set $K$ we can associate the closed geodesic $\gamma$ in the hyperbolic domain $\mathcal{V}-P(F)$ which separates $P(F) \cap K$ from $P(F)-K$. These geodesics cut the domain $\mathcal{V}-P(F)$ in subsets which we will call pieces. 
Lemma 5.3. Let $F: \mathcal{U} \rightarrow \mathcal{V}$ be the extended map defined above. Let $K$ be a small Julia set for $F, P=P(F) \cap K$ and let $\gamma$ be the closed geodesic in $\mathcal{V}-P(F)$ which separates $P$ from $P(F)-K$. There exists $C_{3}$, which depends only on $C_{1}$ and $C_{2}$, so that

- The hyperbolic diameter and length of $\gamma$ in $\mathcal{V}-P(F)$ are $C_{3}$-commensurable to one,

- $\operatorname{dist}(\gamma, P)$ and diam $P$ are $C_{3}$-commensurable,

- The Euclidean diameters of $K, \gamma, P$ and the Euclidean length of $\gamma$ are $C_{3}$-commensurable.

Furthermore, two points in the same piece can be linked by a path in the piece with bounded hyperbolic length in $\mathcal{V}-P(F)$

Proof. Firstly note that, by lemma 5.1 and a priori bounds, $K$ is contained in a larger small Julia set $K^{\prime}$ so that the diameters of $K$ and $K^{\prime}$ are commensurable. Furthermore the diameters of $K$ and $P$ are also commensurable. The hyperbolic length of $\gamma$ is not large because there is unbranched fundamental annulus with definitive modulus for each renormalization. If the Euclidean length of $\gamma$ is small, by the collar lemma (see, e.g., [McM2]), there is a essential annulus with large modulus in $\mathcal{V}-P(F)$, which is impossible, since this planar domain is a $M\left(C_{1}, C_{2}\right)$-uniform domain. Furthermore, by lemma 8.3 the Euclidean diameter of $\gamma$ is commensurable to diam $P$. It is easy to see that the Euclidean length of $\gamma$ is also commensurable to diam $P$. The second statement is consequence of the first one and proposition 8.3. Let $x$ and $y$ be two points in a piece. We can join these points using segments of lines and arcs of geodesics in the boundary of the piece. By the previous items, the sum of the hyperbolic length of the geodesic arcs is bounded. The sum of the Euclidean length of these segments can be clearly bounded by the Euclidean diameter of the piece. On the other hand, by the collar lemma (see, e.g., [McM2]) and previous items, the distance between any point in these segments and the postcritical set is commensurable with the Euclidean diameter of piece. This implies that the sum of the hyperbolic length of these segments is bounded.

We say that a map belongs to $\mathcal{F}(f)$ if the graph of $g$ is contained in $\left\{(x, y): f^{i}(x)=\right.$ $\left.f^{j}(y)\right\}$, for some $i, j \geq 0$. We are going to prove that there are copies of the small Julia sets close to any point in $J(f)$, in any scale:

Proposition 5.7 (Small Julia sets everywhere). Let $f \in P_{n}^{\infty}\left(C_{1}, C_{2}\right)$. There exist $C_{3}\left(C_{1}, C_{2}\right)$ and $C_{4}\left(C_{1}, C_{2}\right)$ with the following property: For any $z$ in $J(f)$ and $\alpha \in(0,1)$ there exists a polynomial like map $g: U \rightarrow V, g \in \mathcal{F}(f)$ so that

- $g \in \mathcal{P}_{n}^{\infty}\left(C_{1}, C_{4}\right)$,

- The diameter of $K(g)$ is $C_{3}$-commensurable to $\alpha \cdot \operatorname{diam}(K(f))$,

- $\operatorname{dist}(z, K(g)) \leq C_{3} \cdot \alpha$.

Proof. The proof is quite similar to the proof in the unimodal case (see [McM2]): Consider a decomposition in ramified coverings of degree two $f=f_{1} \circ \cdots \circ f_{n}$, $f_{i}: U_{i} \rightarrow U_{i+1}$, and the associated extended map $F$ defined in $\mathcal{U}=\{(z, i): z \in$ $U_{i}$ and $\left.1 \leq i \leq n\right\}$ by

$$
F(z, i)=\left(f_{i}(z), i+1 \bmod n\right)
$$

Define the postcritical set of $F$ by $P(F)=\cup_{i} F^{i}(P(f))$. If $\|\cdot\|_{\mathcal{V}-P(F)}$ denote the hyperbolic metric on $\mathcal{V}-P(F)$ (extended to $\infty$ on $P(F)$ ) then

$$
\left\|F^{\prime}(x) \cdot v\right\|_{\mathcal{V}-P(F)}>\|v\|_{\mathcal{V}-P(F)},
$$


with $v$ in the tangent space of $x$, since $F^{-1}(P(F)) \supset P(F)$. Let $v_{k}=D F^{k}(x) \cdot v$, with $x \in J(F)$. Then

$$
\left\|v_{k}\right\|_{\mathcal{V}-P(F)}<\left\|v_{k+1}\right\|_{\mathcal{V}-P(F)} \rightarrow \infty
$$

since $\cup_{n} F^{-n}(P(F))$ is dense in $J(F)$. For $x \in J(F)$, select a vector $v$ in its tangent space so that $|v|=\alpha$. There are 3 cases:

- $\operatorname{dist}(x, P(F)) \leq \alpha$,

- $\operatorname{dist}(x, P(F))>\alpha$ and there exists $k$ such that $\left\|v_{k}\right\|_{\mathcal{V}-P(F)} \leq \epsilon$ and $\left\|v_{k+1}\right\|_{\mathcal{V}-P(F)} \geq 1 / \epsilon$

- $\operatorname{dist}(x, P(F))>\alpha$ and $\left\|v_{k}\right\|_{\mathcal{V}-P(F)} \sim 1$.

Here $\epsilon$ is sufficiently small so that the McMullen's argument([McM2]) works in the second case. The first and second cases are more easy and we will omit the proof. For details, see [McM2]. Assume the last situation. In particular $\|v\|_{\mathcal{V}-P(F)}<1$, because $\mathcal{V}-P(F)$ is a $M\left(C_{1}, C_{2}\right)$-uniform domain.

Consider the piece, defined by closed geodesics, which contains $F^{k}(x)$. Let $\gamma_{j}$ be the exterior geodesic and let $\gamma_{1, j+1}, \ldots, \gamma_{i, j+1}$ be the interior boundary geodesics. Denote by $P(i, j+1)$ the subset of the postcritical set bounded by $\gamma_{i, j+1}$. Select $\ell$ minimal so that we can do the univalent pullback of the domain $V$ bounded by $\gamma_{j}$ along the inverse orbit $F^{k}(x), F^{k-1}(x), \ldots, F^{\ell}(x)$. This means there exists a simply connected domain $V^{\prime}$ satisfying

- $F^{\ell}(x) \in V^{\prime}$.

- The map $F^{k-\ell}$ is univalent in $V^{\prime}$ and moreover $F^{k-\ell}\left(V^{\prime}\right)=V$.

- The domain $V^{\prime}$ contains a critical value of $F$.

Denote by $V_{i}$ the domain bounded by $\gamma_{j+1, i}$ and let $V_{i}^{\prime}$ be the corresponding domain in $V^{\prime}$. Let $\tilde{V}=F^{-1}\left(V^{\prime}\right)$ Then

$$
g=F^{k-\ell+1}: \tilde{V} \rightarrow V
$$

is a proper map of degree two. Since the postcritical set is contained in $\cup_{i} V_{i}$, the critical value in $V^{\prime}$ is contained in some $V_{i_{0}}^{\prime}$, for some $i_{0}$. Choose an arbitrary $V_{i_{1}}$, $i_{1} \neq i_{0}$. Let $\beta_{1}$ and $\beta_{2}$ be two paths inside the piece which contains $F^{k}(x)$ so that:

- The initial point of both is $F^{k}(x)$.

- The end point of both is a point in $\gamma_{j, i_{1}}$.

- The Jordan curve defined by $\beta_{1}$ and $\beta_{2}$ is not homotopic to a constant curve in $V-F^{k-\ell}(v)$.

- The hyperbolic diameter of $\beta_{i}$ on $\mathcal{V}-P(F)$ is bounded.

Let $\tilde{\beta}_{1} \cup \tilde{U}^{1}$ and $\tilde{\beta}_{2} \cup \tilde{V}^{2}$ be lifts with respect to $g$ of the simply connected sets $\beta_{1} \cup V_{i_{1}}$ and $\beta_{2} \cup V_{i_{1}}$ so that $\tilde{\beta}_{i}$ is an arc whose initial point is $F^{\ell-1}(x)$. Note that $\tilde{V}^{1}$ and $\tilde{V}^{2}$ are disjoint and one of them, say $\tilde{V}^{1}$, does not intersect the postcritical set of $F$. So all inverse branches of $F$ are well defined on $\tilde{\beta}_{1} \cup \tilde{V}^{1}$. So let $h$ be the inverse branch of $F^{k}$, defined in $\beta_{1} \cup V_{i_{1}}$ so that $h\left(F^{k}(x)\right)=x$. Since $\left\|v_{k}\right\|_{\mathcal{V}-P(F)} \sim 1$ and $\beta_{1} \cup \gamma_{j+1, i_{1}}$ has bounded hyperbolic diameter in $\mathcal{V}-P(F)$, we obtain, by corollary 8.4 ,

$$
|D h(z)| \sim \frac{\left|v_{0}\right|}{\left|v_{k}\right|}
$$

for all $z \in \beta_{1} \cup \gamma_{j+1, i_{1}}$. By the maximum principle the same distortion control holds in $U_{i_{1}}$. There exists a small Julia set $K$ inside $V_{i_{1}}$ whose diameter is commensurable to $\operatorname{diam} \gamma_{j+1, i_{1}} \sim\left|v_{k}\right|$. By $1 / 4$-Koebe lemma (use that $\operatorname{dist}\left(\gamma_{j+1, i_{1}}, P\left(j+1, i_{1}\right)\right) \geq$ 
$\left.C \operatorname{diamP}\left(j+1, i_{1}\right)\right)$ and the above distortion control, the set $h(K)$ has diameter commensurable with $\left|v_{0}\right|=\alpha$. Moreover $\operatorname{dist}(x, h(K)) \leq C \alpha$, which proves the proposition.

Corollary 5.1. The following holds:

- A polynomial like map in $\mathcal{P}_{n}^{\infty}\left(C_{1}\right)$ does not support invariant line fields in it Julia set,

- The hybrid class on $\mathcal{P}_{n}\left(C_{2}\right)$ is continuous at points in $\mathcal{P}_{n}^{\infty}\left(C_{1}, C_{2}\right)$,

- The set $\mathcal{P}_{n}^{\infty}\left(C_{1}, C_{2}\right)$ is compact.

Proof. Suppose, by contradiction, there exists an invariant line field $\mu$ supported on the Julia set $K(f)$ on $f \in \mathcal{P}_{n}^{\infty}\left(C_{1}\right)$. Select an almost continuity point $x \in J(f)$ to $\mu$. There are polynomial like maps, with definitive modulus, in all scales around $x$, which preserves $\mu$. After an affine conjugacy, we can assume that a subsequence of these polynomial like maps converge to a polynomial like map which preserves a straight line field, which is a contradiction. The second statement is consequence of the lemma in pg. 313 of $[\mathrm{DH}]$. The last statement is an immediate consequence of the first ones.

W. Shen [Sh1] proved that any real polynomial with real critical points does not support invariant line fields in it Julia set. The above situation is not included, in general, in Shen's result, but we can expect the same result (probably with a very similar proof) for compositions of real polynomials with real critical points.

\section{HYBRID CONJUGACY}

As in the unimodal case (see $[\mathrm{Su}]$ and $[\mathrm{dMvS}])$ and bimodal case $([\mathrm{H} 1])$, real maps with same bounded combinatorics are hybrid conjugated. To be more precise:

Theorem 1. Two real polynomial-like maps of type $n, f$ and $\tilde{f}$, infinitely renormalizable map with same bounded combinatorics are hybrid conjugated.

Let $f$ and $\tilde{f}$ be multimodal maps of type $n$ with decompositions $\left(f_{1}, \ldots, f_{n}\right)$ and $\left(\tilde{f}_{1}, \ldots, \tilde{f}_{n}\right)$. If $c_{i}$ (resp. $\left.\tilde{c}_{i}\right)$ is the critical point of $f_{i}$ (resp. $\tilde{f}_{i}$ ), define $v_{i}=$ $f_{n} \circ \cdots \circ f_{i}\left(c_{i}\right)\left(\operatorname{resp} . \tilde{v}_{i}=\tilde{f}_{n} \circ \cdots \circ \tilde{f}_{i}\left(\tilde{c}_{i}\right)\right.$.

Lemma 6.1 (Lifts exist). Let $f: U_{0} \rightarrow U_{n}$ and $\tilde{f}: \tilde{U}_{0} \rightarrow \tilde{U}_{n}$ be real polynomial-like maps of type $n$ with the same inner itinerary and such that $v_{i}<v_{j}$ iff $\tilde{v}_{i}<\tilde{v}_{j}$. Then the following holds: For any continuous bijection $H_{n}: U_{n} \rightarrow \tilde{U}_{n}$, real in the real line and increasing in $\mathbb{R}$, such that $H(f(C(f)))=\tilde{f}(C(\tilde{f}))$, there exists a continuous bijection $H_{0}: U_{0} \rightarrow \tilde{U}_{0}$, real in the real line and increasing in $\mathbb{R}$, such that $H_{n} \circ f=\tilde{f} \circ \tilde{H}_{0}$.

Proof. We will define, by induction, homeomorphisms $H_{i}: U_{i} \rightarrow \tilde{U}_{i}$, real in the real line and increasing, such that $H_{i+1} \circ \tilde{f}_{i}=f_{i} \circ H_{i}$. Assume that we have defined $H_{i}$. We claim that $H_{i}\left(f_{i-1}\left(c_{i-1}\right)\right)=\tilde{f}_{i-1}\left(\tilde{c}_{i-1}\right)$. Indeed, consider $A_{i}=\left(f_{n} \circ \cdots \circ\right.$ $\left.f_{i}\right)^{-1}\left(v_{i-1}\right)$ and $\tilde{A}_{i}=\left(\tilde{f}_{n} \circ \cdots \circ \tilde{f}_{i}\right)^{-1}\left(\tilde{v}_{i-1}\right)$. Since $H_{n} \circ f_{n} \circ \cdots \circ f_{i}=\tilde{f}_{n} \circ \cdots \circ \tilde{f}_{i} \circ H_{i}$ and $H_{n}\left(v_{i-1}\right)=\tilde{v}_{i-1}$, we have $H_{i}\left(A_{i}\right)=\tilde{A}_{i}$. Since $H_{i}$ is increasing, it suffice to show that if $f_{i-1}\left(c_{i-1}\right)$ is the $j$-th point in $A_{i}$, with respect to the order in the real line, then $\tilde{f}_{i-1}\left(\tilde{c}_{i-1}\right)$ is also the $j$-th point in $\tilde{A}_{i}$. But this follows of lemma 2.3.3, since $f_{i-1}\left(c_{i-1}\right)$ and $\tilde{f}_{i-1}\left(\tilde{c}_{i-1}\right)$ have the same inner itinerary. 
Now we can find a homeomorphism $H_{i-1}: U_{i-1} \rightarrow \tilde{U}_{i-1}$, real in the real line and increasing such that

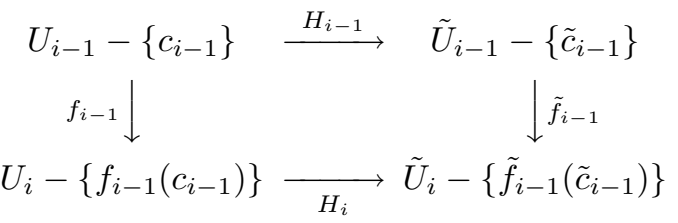

commutes, which proves the lemma.

Lemma 6.2. If $f$ and $\tilde{f}$ are as in Theorem 1, then there exists a quasiconformal map $h: \mathbb{C} \rightarrow \mathbb{C}$, which is real in the real line and increasing such that $h(P(f))=$ $P(\tilde{f})$ and $h \circ f=\tilde{f} \circ h$ in $P(f)$.

Proof. This lemma is consequence of the bounded geometry (see proposition 5.1). For details see $[\mathrm{Su}]$ or $[\mathrm{dMvS}]($ last chapter).

Proof of Theorem 1. Replacing $U, \tilde{U}, V$ and $\tilde{V}$ by smaller domains, we can assume that the boundary of these domains are quasicircles. Using similar arguments as in lemma 4.4, we can construct a quasiconformal map $h_{1}: \bar{V}-U \rightarrow \overline{\tilde{V}}-\tilde{U}$ which conjugates $f$ and $\tilde{f}$ in $\partial U$. Since the maps $f$ and $\tilde{f}$ are real, we can assume that $h_{1}$ is symmetric with respect to the real line. Let $h_{2}$ be a symmetric quasiconformal map which conjugates $f$ and $\tilde{f}$ in the postcritical set. Construct a $C$-quasiconformal map $H_{0}: V \rightarrow \tilde{V}$, for some $C$, which is symmetric, increasing in the real line, equal to $h_{1}$ in $\bar{V}-U$ and equal to $h_{2}$ in a neighborhood of $I$. As $f$ and $\tilde{f}$ have the same combinatorial type, the relative positions of $v_{i}$ and $\tilde{v}_{i}$ are the same. Thus we can use lemma 6.1. Furthermore $f$ and $\tilde{f}$ has the same inner itinerary. Define inductively $H_{j}: V \rightarrow \tilde{V}$, a $C$-quasiconformal map symmetric, increasing and such that $H_{j} \circ f=f \circ H_{j+1}$. Note that $H_{j}$ is $C$-quasiconformal conjugacy in the postcritical set and $V-f^{-(j-1)}(U)$. Moreover $H_{j}=H_{j+1}$ in $V-f^{-(j-1)}(U)$. Since $K(f)$ has empty interior, the sequence $H_{j}$ has an unique limit $H$, which is a conjugacy between $f$ and $\tilde{f}$. Indeed $H$ is a hybrid conjugacy by lemma 5.1.

Theorem 2. The set $\operatorname{Pol}_{n}^{\infty}(C)$ is a Cantor set.

Proof. Let $\Sigma=\Sigma_{C}^{n}$ be the finite set of primitive, transitive m.c.d. with combinatorics bounded by $C$. By lemma 2.3 , for any infinity sequence $\left(\sigma_{1}, \sigma_{2}, \ldots\right)$, with $\sigma_{i} \in \Sigma$, there exists an infinitely renormalizable real polynomial map of type $n$ with this combinatorial type. By the previous theorem, two real polynomial maps of type $n$ with same combinatorics are hybrid conjugated and so affine conjugated, since they are polynomials. The boundary points of $I$ must be a fixed by this affine map. Thus the conjugacy must be the identity. Let $\Pi: \Sigma^{\mathbb{N}} \rightarrow A_{n}$ be the application which maps each sequence $\alpha=\left(\sigma_{1}, \sigma_{2}, \ldots\right)$ in the unique real polynomial map $p_{\alpha}$ of type $n$ with this combinatorial type. If $\alpha_{i} \in \Sigma^{\mathbb{N}}$ is a sequence which converges to $\alpha$, then any accumulation point $p$ of the sequence $p_{\alpha_{i}}$ is a real infinitely renormalizable polynomial of type $n$ with combinatorics $\alpha$. So $p=p_{\alpha}$. Hence $\Pi$ is a homeomorphism between the Cantor set $\Sigma^{\mathbb{N}}$ and $\operatorname{Pol}_{n}^{\infty}(C)$.

A natural conjecture, in view of M. Lyubich results in the quadratic family, is

Conjecture 1. The set $\operatorname{Pol}_{n}^{\infty}(C)$ has zero n-dimensional Lebesgue measure. 


\section{Contraction of REnORMalization}

7.1. Towers. Using McMullen arguments with towers ([McM2]) we will prove the contraction of the renormalization operator in the hybrid classes of infinitely renormalizable maps with bounded combinatorics. The main point here is that the Julia set of bi-infinite towers is dense in the complex plane. Together the small Julia sets everywhere Theorem, we will obtain the contraction. Apart McMullen's work, the renormalization theory in $[\mathrm{SN}]$ had some influence in the exposition below.

Definition 7.1. A bi-infinity tower $\mathbf{f}=<f_{i}>_{i \in \mathbb{Z}}$ of type $n$ with parameters $C_{1}$, $C_{2}$ and $k$ is a family of polynomial-like maps $f_{i}: U_{i} \rightarrow V_{i}$ of type $n, i \in \mathbb{Z}$, such that

- The maps $f_{i}$ belongs to $\mathcal{P}_{n}^{\infty}\left(C_{1}, C_{2}\right)$;

- For any $i \in \mathbb{Z}$, there exists $a \leq k$ so that $R^{a}\left(f_{i-1}\right)=f_{i}$ (as germs of functions).

if furthermore we assume that

- If $j \geq i$ then $V_{j}$ is contained in $U_{i}$.

we say that $\mathbf{f}$ is a fine tower. Denote by $\mathcal{T}_{n}\left(C_{1}, k, C_{2}\right)$ (resp. $\mathcal{T}_{n}^{\text {fine }}\left(C_{1}, k, C_{2}\right)$ ) the set of bi-infinity towers (resp. fine towers) with parameters $C_{1}, k, C_{2}$.

We say that a bi-infinite tower $\mathbf{g}$ is a restriction of other tower $\mathbf{f}$ if there exists an increasing function $j: \mathbb{Z} \rightarrow \mathbb{Z}$ so that the germ $g_{i}$ coincides with the germ $f_{j(i)}$. The following lemma is easy to prove:

Proposition 7.1. For any tower $f \in \mathcal{T}_{n}\left(C_{1}, 1, C_{2}\right)$, there exists a restriction $g \in$ $\mathcal{T}_{n}^{\text {fine }}\left(C_{1}, k, C_{2}\right)$, with $k=k\left(C_{1}, C_{2}\right)$.

McMullen([McM2]) supply the set of towers with the following sequential convergence: We say that the sequence of towers $\mathbf{f}_{n}$ converges to tower $\mathbf{f}_{\infty}$ if

- For any $i \in \mathbb{Z}$ there exists $a$ so that $f_{i+1, n}=R^{a}\left(f_{i, n}\right)$, for large $n$;

- $f_{i, n}$ converges to $f_{i, \infty}$.

Proposition 7.2. The sets $\mathcal{T}_{n}\left(C_{1}, k, C_{2}\right)$ and $\mathcal{T}_{n}^{\text {fine }}\left(C_{1}, k, C_{2}\right)$ are compact.

Select an arbitrary $j_{1} \in \mathbb{Z}$. Then $A_{j}=V_{j}-U_{j}, j_{0}<j<j_{1}$, are disjoint essential annulus in $V_{j_{0}}-K\left(f_{j_{1}}\right)$. Because $\operatorname{diam} K\left(f_{j_{1}}\right)>0$ and $\bmod A_{j}>m\left(C_{1}\right)$, $\mathbb{C}=\cup_{j} V_{j}$. We say that a line field $\mu$ is invariant by the tower $\mathbf{f}$ if $\mu$ is invariant for each $f_{i}, i \in \mathbb{Z}$.

Proposition 7.3 (Constructing bi-infinite towers). Let $f_{j, i}: U_{j, i} \rightarrow V_{j, i}$; with $i \in \mathbb{N}$ and $|j| \leq j(i), j(i) \rightarrow_{i} \infty$; be polynomial-like maps in $\mathcal{P}_{n}^{\infty}\left(C_{1}, C_{2}\right)$ such that there exists $k$ satisfying

$$
f_{j+1, i}=R^{a}\left(f_{j, i}\right), \text { where } a \leq k .
$$

Then we can select a subsequence $i_{k}$ such that $f_{j, i_{k}} \rightarrow f_{j, \infty}$, where $\mathbf{f}_{\infty}=<f_{j, \infty}>$ is a tower in $\mathcal{T}_{n}\left(C_{1}, k, C_{2}\right)$. If $V_{j+1, i} \subset U_{j, i}$, then $\mathbf{f}_{\infty} \in \mathcal{T}_{n}^{\text {fine }}\left(C_{1}, k, C_{2}\right)$.

Proposition 7.4 (Constructing conjugacies). Let

$$
f_{j, i}: U_{j, i} \rightarrow V_{j, i} \text { and } \tilde{f}_{j, i}: \tilde{U}_{j, i} \rightarrow \tilde{V}_{j, i}
$$

with $|j| \leq j(i)$, be as in the previous lemma. Let $h_{i}: \mathbb{C} \rightarrow \mathbb{C}$ be $C$-quasiconformal maps so that:

- $h_{i}$ is a hybrid conjugacy between $f_{j, i}$ and $\tilde{f}_{j, i}$, for $|j| \leq j(i)$, 
- $h_{i}\left(U_{j, i}\right)=\tilde{U}_{j, i}$.

Then we can select a subsequence $i_{\ell}$ such that

$$
<f_{j, i_{\ell}}>_{|j| \leq j\left(i_{\ell}\right)} \text { and }<\tilde{f}_{j, i_{\ell}}>_{|j| \leq j\left(i_{\ell}\right)}
$$

converge to bi-infinite towers $\mathbf{f}_{\infty}$ and $\tilde{\mathbf{f}}_{\infty}$ and $h_{i_{\ell}}$ converges to a conjugacy between these towers.

Fix a bi-infinite fine tower $f=<f_{i}>_{i \in \mathbb{Z}}$ in $\mathcal{T}_{n}^{\text {fine }}\left(C_{1}, k, C_{2}\right)$. Denote by $P_{i}$ the postcritical set of $f_{i}$. Define $Q_{i}=f_{i}^{-1}\left(P_{i}\right)$. Let $P=\cup_{i} P_{i}$.

Lemma 7.1. Suppose that $z \in U_{i}$ with $f_{i}(z) \in V_{i}-P_{i}$. Then

$$
\left\|f_{i}^{\prime}(z)\right\|_{\mathbb{C}-P, \mathbb{C}-P} \geq 1
$$

Proof. Since $f_{i}: U_{i}-Q_{i} \rightarrow V_{i}-P_{i}$ is a covering map

$$
\left\|f_{i}^{\prime}(z)\right\|_{U_{i}-Q_{i}, V_{i}-P_{i}}=1 \text {, }
$$

and furthermore

$$
\left\|i_{U_{i}-Q_{i}, V_{i}-P_{i}}\right\|_{U_{i}-Q_{i}, V_{i}-P_{i}}<1
$$

we obtain

$$
\left\|f^{\prime}(z)\right\|_{V_{i}-P_{i}, V_{i}-P_{i}}>1 \text {. }
$$

Since the hyperbolic metric in $V_{i}-P_{i}$ converges to the hyperbolic metric in $\mathbb{C}-P$, we obtain the lemma.

Lemma 7.2 (Strict expansion([McM2])). There exists $\lambda=\lambda\left(C_{1}, C_{2}\right)>1$ with the following property: Let $z \in U_{i}$ be such that $f_{i}(z) \in V_{i}-U_{i}$. Then

$$
\left\|f_{i}^{\prime}(z)\right\|_{\mathbb{C}-P} \geq \lambda
$$

Proof. We sketch the McMullen's proof: Consider $j<i$. We have $f_{i}=f_{j}^{a}$, for some $a>0$. so

$$
\left\|f_{i}^{\prime}(z)\right\|_{f_{j}^{-a}\left(V_{j}-P_{j}\right), V_{j}-P_{j}}=1 .
$$

Since $z \in f_{i}^{-1}\left(V_{i}-U_{i}\right)$, by the inclusion contraction lemma (proposition 4.9 in [McM2]):

$$
\left\|i_{f_{j}^{-a}\left(V_{j}-P_{j}\right), V_{j}-P_{j}}\right\|_{U_{i}-Q_{i}, V_{j}-P_{j}} \leq C(D)<1
$$

It follows that $\left\|f_{i}^{\prime}(z)\right\|_{V_{j}-P_{j}} \geq \lambda(D)$. Now it is suffice to observe that $\rho_{V_{j}-P_{j}} \rightarrow$ $\rho_{\mathbb{C}-P}$.

Let $i<j$ be such that $z_{0} \in U_{j}-J\left(f_{i}\right)$. Consider $t_{\ell}$, with $i \leq \ell \leq j$, such that

$$
f_{\ell}^{t_{\ell}} \circ f_{\ell+1}^{t_{\ell+1}} \circ \cdots \circ f_{j-1}^{t_{j-1}} \circ f_{j}^{t_{j}}\left(z_{0}\right) \in V_{\ell}-U_{\ell}
$$

Let $\tilde{A}$ be a simply connected domain in $V_{i}-P_{i}$ which contains $\tilde{z_{0}}=f_{\ell}^{t_{\ell}-1} \circ f_{\ell+1}^{t_{\ell+1}} \circ$ $\cdots \circ f_{j-1}^{t_{j-1}} \circ f_{j}^{t_{j}}\left(z_{0}\right)$. Note that $f_{\ell}^{t_{\ell}-1} \circ f_{\ell+1}^{t_{\ell+1}} \circ \cdots \circ f_{j-1}^{t_{j-1}} \circ f_{j}^{t_{j}}=f_{i}^{a-1}$, for some $a$, and so there exists a simply connected domain $A$ such that $z_{0} \in A$ and $f_{i}^{a-1}$ restricts to $A$ is an univalent map whose image is $\tilde{A}$, since $f_{i}^{a-1}: f_{i}^{-(a-1)}\left(V_{i}-P_{i}\right) \rightarrow V_{i}-P_{i}$ is a covering map.

The following result is the main key in proof of density of the Julia set of a tower and, in fact, it is an old trick: to study a dynamical system with singularities, it 
is useful to find expansion and distortion control in a dynamics induced by the original dynamics:

Proposition 7.5. In the conditions described above, the following holds:

(1) Uniform expansion: We have:

$$
\left\|D\left(f_{i}^{a}\right)\left(z_{0}\right)\right\|_{\mathbb{C}-P} \geq \lambda^{j-i}
$$

(2) Distortion control: If $\operatorname{diam}_{V_{i}-P_{i}}(\tilde{A}) \leq D$ then

$$
\frac{1}{C(D)} \leq \frac{\left\|D\left(f_{i}^{a-1}\right)\left(z_{1}\right)\right\|_{\mathbb{C}-P}}{\left\|D\left(f_{i}^{a-1}\right)\left(z_{2}\right)\right\|_{\mathbb{C}-P}} \leq C(D),
$$

for $z_{1}, z_{2} \in A$.

Proof. The first statement is an immediate consequence of lemmas 7.1 and 7.2. For the second statement, note that $f_{i}^{a-1}(z) \in f_{i}^{-1}\left(V_{i}-U_{i}\right)$. The map

$$
f_{i}^{a-1}: f_{i}^{-(a-1)}\left(V_{i}-P_{i}\right) \rightarrow V_{i}-P_{i}
$$

is a covering map, with $f_{i}^{-(a-1)}\left(V_{i}-P_{i}\right) \subset V_{i}-P_{i}$, thus we can apply corollary 8.4 (twice) to obtain 2 (Note that $V_{i}-P_{i}$ and $\mathbb{C}-P$ are $M\left(C_{1}, C_{2}\right)$-uniform domains).

Our distortion control is stronger than the similar statement in McMullen book because we are using that the geometry of postcritical set is bounded in more points in the proof.

Corollary 7.1. Let $\mathbf{f}$ be a tower in $\mathcal{T}_{n}\left(C_{1}, k, C_{2}\right)$. Then the set $J(\mathbf{f})=\cup_{i \in \mathbb{Z}} J\left(f_{i}\right)$ is dense in $\mathbb{C}$.

Proof. By Proposition 7.1, we can assume that $\mathbf{f}$ is fine. Let $z$ be a complex number which is not contained in $J(\mathbf{f})$. Then $z$ is not in $J\left(f_{k}\right)$ for sufficiently small $k$. Let $k_{0}$ be maximal such that $z \in U_{k_{0}}$. For each small $k>k_{0}$ let $a(k)$ be minimal so that $f^{a(k)}(z) \in f_{k}^{-1}\left(V_{k}-U_{k}\right)$ and let $\gamma_{k}$ be the minimal geodesic between $f_{k}^{a(k)}(z)$ and $J\left(f_{k}\right)$ in $V_{k}-P_{k}$. The hyperbolic length of $\gamma_{k}$ is smaller than $D$, where $D$ is as in lemma 5.6, since $f_{k}^{-1}\left(P_{k}\right) \subset J\left(f_{k}\right)$. By the previous lemma, the length of the lift $\tilde{\gamma}_{k}=f_{k}^{-a(k)} \gamma_{k}$ in the hyperbolic metric of $\mathbb{C}-P$ goes exponentially fast to zero, when $-k$ goes to infinity. Since the end points of $\tilde{\gamma}_{k}$ are $z$ and a point in the Julia set, we finished the proof (recall that the hyperbolic metric in $\mathbb{C}-P$ is $\rho(x)|d z|$, where $\rho(x)$ is comparable with $1 / \operatorname{dist}(x, P))$.

Definition 7.2. Let $\Lambda \subset \mathbb{C}$. We say that $z$ is a $\delta$-deep point of $\Lambda$ if there exists $C$ so that, for $\tilde{z} \in \mathbb{C}$, $\operatorname{dist}(\Lambda, \tilde{z}) \leq C \operatorname{dist}(z, \tilde{z})^{1+\delta}$;

We can use a similar argument as in [McM2] to infinite towers $\mathbf{f}=<f_{i}>_{i \in \mathbb{N}}$ to obtain statements as lemmas 7.1 and 7.2 and proposition 7.5 , replacing the hyperbolic manifold $\mathbb{C}-P$ by $V_{0}-P\left(f_{0}\right)$. This is useful to prove:

Corollary 7.2. The critical point 0 of $f \in P_{n}^{\infty}\left(C_{1}, C_{2}\right)$ is a $\delta\left(C_{1}, C_{2}\right)$-deep point of $J(f)$.

Proof. By Proposition 5.5.6, the renormalizations have extensions $R^{k} f: U^{k} \rightarrow$ $V^{k} \in P_{n}^{\infty}\left(C, C_{2}\right)$. Let $x$ be a complex number close to 0 . We can find $k>-C \ln \| x \mid$, 
with $C=C\left(C_{1}, C_{2}\right)$, such that $x \in U^{k}$. We can assume that $x$ is not in $J(f)$. By the same argument as in the previous corollary

$$
\operatorname{dist}_{V-P(f)}(x, J(f)) \leq C \lambda^{C k} \leq C|x|^{\delta}
$$

Since the hyperbolic metric in $V-P(f)$ is $\rho(x)|d z|$, where $\rho(x)$ is commensurable with $1 / \operatorname{dist}(x, P(f) \cup \partial V)$, it is easy to see that the Euclidean length of the minimal hyperbolic geodesic between $x$ and $J(f)$ is bounded by $C|x|^{1+\delta}$.

Corollary 7.3 (Rigidity). The towers in $\mathcal{T}\left(C_{1}, k, C_{2}\right)$ does not support non trivial invariant Beltrami fields.

Proof. Let $\mu$ be an invariant line field to the tower $\mathbf{f}$. Because $K\left(f_{i}\right)$ does not support invariant line fields, it is possible select a point $z_{0} \in \mathbb{C}-K(\mathbf{f})$ where $\mu$ is almost continuous. This means

$$
\lim _{\delta \rightarrow 0} \frac{\ell\left(\left\{z:\left|z-z_{0}\right|<\delta \text { and }\left|\mu(z)-\mu\left(z_{0}\right)\right| \leq \epsilon\right\}\right)}{\ell\left(\left\{z:\left|z-z_{0}\right|<\delta\right\}\right)}=1 .
$$

Here $\ell$ is the Lebesgue measure in $\mathbb{C}$. Since $J(\mathbf{f})$ is dense and by small Julia sets everywhere theorem, for any $\alpha>0$ there exists a polynomial like map $g_{\alpha}: V_{1}^{\alpha} \rightarrow V_{2}^{i}$ so that:

- The map $g_{\alpha}: V_{1}^{\alpha} \rightarrow V_{2}^{\alpha}$ belongs to $\mathcal{F}\left(f_{i}\right)$, for some $i$ (indeed, for any $i$ small enough);

- The map $g_{\alpha}: V_{1}^{\alpha} \rightarrow V_{2}^{\alpha}$ belongs to $\mathcal{P}_{n}\left(C\left(C_{1}, C_{2}\right)\right)$;

- $\operatorname{diam}\left(J\left(g_{\alpha}\right)\right) \sim \alpha$;

- $\operatorname{dist}\left(z_{0}, J\left(g_{\alpha}\right)\right) \leq C\left(C_{1}, C_{2}\right) \cdot \alpha$.

Since $\mu$ is invariant by these maps, normalizing $g_{\alpha}$ so that $\operatorname{diam}\left(J\left(g_{\alpha}\right)\right)=1$, we can select a subsequence which converges to a polynomial like map which preserves a straight line field. This is impossible.

Let $\sigma=\left(\sigma_{0}, \sigma_{1}, \ldots\right)$ be a sequence of m.c.d. We will denote by $R^{k}(\sigma)$ the bi-infinite sequence

$$
\left(\ldots, \tilde{\sigma}_{-1}, \tilde{\sigma}_{0}, \tilde{\sigma}_{1}, \ldots\right)
$$

where $\tilde{\sigma}_{i}=\sigma_{i+k}$ for $i \geq-k$. Fill the other positions in the sequence in an arbitrary way (we are interested in convergent subsequences of $R^{k}(\sigma)$ when $k \rightarrow \infty$ in the space of bi-infinite sequences. Thus the other positions are not important for us).

Corollary 7.4. There exists an unique bi-infinite tower $\mathbf{g}_{\sigma}$ in $\mathcal{T}_{n}\left(C, 1, C_{2}\right)$ with C-bounded combinatorics

$$
\sigma=\left(\ldots, \sigma_{-2}, \sigma_{-1}, \sigma_{0}, \sigma_{1}, \sigma_{2}, \ldots\right)
$$

Here uniqueness means that if $\mathbf{g}$ and $\tilde{\mathbf{g}}$ are bi-infinite towers with same combinatorics then the germs $g_{i}$ and $\tilde{g}_{i}$ are the same (up to an affine conjugacy). Furthermore there exists $C_{1}(C)$ such that the germ $g_{i}$ has a representation $g_{i}: U^{i} \rightarrow V^{i}$ which belongs to $\mathcal{P}_{n}^{\infty}\left(C, C_{1}\right)$. Notice that $C_{1}$ does not depend on $\sigma$.

Proof. Existence: Select a real infinitely renormalizable polynomial $p_{0}$ of type $n$ with combinatorics $\tilde{\sigma}=\left(\tilde{\sigma}_{0}, \tilde{\sigma}_{1}, \ldots\right)$ so that for any $C$-bounded combinatorics there exists a sequence $k_{i}$ satisfying $R^{k_{i}}(\tilde{\sigma}) \rightarrow_{k} \sigma$. Using the complex bounds, select, for renormalizations deep enough, polynomial like representations in $\mathcal{P}_{n}^{\infty}(C, C(f))$. Then the finite tower $\left\langle g_{j, i}\right\rangle_{|j| \leq k_{i}}$ defined by

$$
g_{j, i}=R^{k_{i}+j} p_{0}
$$


has a subsequence which converges to a bi-infinite tower in $\mathcal{T}_{n}\left(C, 1, C_{1}(f)\right)$ with combinatorics $\sigma$.

Uniqueness: Let $\mathbf{f}$ and $\mathbf{g}$ be bi-infinity towers in $\mathcal{T}_{n}\left(C_{1}, 1, C_{2}\right)$. Since $g_{i}: U_{i}^{g} \rightarrow$ $V_{i}^{g}$ and $f_{i}: U_{i}^{f} \rightarrow V_{i}^{f}$ have the same combinatorics, there exists one $C\left(C_{1}, C_{2}\right)$ quasiconformal map $\phi_{i}: \mathbb{C} \rightarrow \mathbb{C}$ which maps $U_{i}^{f}$ in $U_{i}^{g}$ and it is a conjugacy between $f_{i}$ and $g_{i}$ in $U_{i}^{f}$. When $i \rightarrow-\infty$ we have $V_{i}^{f} \rightarrow \mathbb{C}$. Thus $\phi_{i}$ admits a convergent subsequence to some quasiconformal map $\phi: \mathbb{C} \rightarrow \mathbb{C}$. This map is a conjugacy between the tower $\mathbf{f}$ and the tower $\tilde{\mathbf{g}}$, where $\tilde{g}_{i}$ is equal to $g_{i}$ restricts to $\phi\left(U_{i}^{f}\right)$. Since the Beltrami field $\frac{\bar{\partial} \phi}{\partial \phi}$ is invariant by the tower $\mathbf{f}$, the rigidity of towers implies that $\phi$ is conformal. Thus, up to affine maps, $\phi$ is the identity.

Let $V$ be a domain in $\mathbb{C}$. denote by $B(V)$ the Banach space of the holomorphic functions defined in $V$ and with a continuous extension to $\bar{V}$. Denote by $\mathcal{A}_{n}^{\infty}(C)$ the set of germs $g$ in some level (and hence in the level 0 ) of a bi-infinite tower in $\mathcal{T}_{n}\left(C, 1, C_{1}\right)$, for some $C_{1}$. Apart the rate of contraction, the main theorem was reduced to

Theorem 3 (Contraction of renormalization). There exists $\delta=\delta\left(C_{1}\right)$ with the following property: For any $\epsilon>0$ there exists $j_{0}=j_{0}\left(C_{1}, C_{2}\right)$ so that if $f \in \mathcal{A}_{n}^{\infty}(C)$ and $g \in \mathcal{P}_{n}^{\infty}\left(C_{1}, C_{2}\right)$ are polynomial like maps with same combinatorics then, for $j \geq j_{0}$ :

- The germ $R^{j}(g)$ belongs to $\mathcal{P}_{n}^{\infty}\left(C_{1}, C_{3}\right)$. Here $C_{3}=C_{3}\left(C_{1}\right)$.

- The renormalizations $R^{j}(f), R^{j}(g)$ belong to $B\left(\delta-K\left(R^{j}(f)\right)\right)$, for $j \geq j_{0}$ and

$$
\left|R^{j}(f)(z)-R^{j}(g)(z)\right| \leq \epsilon
$$

for $z \in \delta-K\left(R^{j}(f)\right)$.

Proof. Since $f \in \mathcal{P}_{n}^{\infty}\left(C_{1}, C\left(C_{1}\right)\right)$ there exists a $K\left(C_{2}\right)$-quasiconformal conjugacy $\phi$ between $f$ and $g$. Normalizing $R^{j}(f)$ and $R^{j}(g)$ so that $R^{j}(f)(0)=1=R^{j}(g)(0)$, we obtain quasiconformal conjugacies $\phi_{j}$ so that $\phi_{j}(0)=0$ and $\phi_{j}(1)=1$. We claim that $\phi_{j}$ converges uniformly in compact sets to identity. Indeed, suppose by contradiction that there exist sequences of maps $f_{\ell}$ and $g_{\ell}, f_{\ell} \in \mathcal{A}_{n}^{\infty}(C)$ and $g_{\ell} \in$ $\mathcal{P}_{n}^{\infty}\left(C_{1}, C_{2}\right)$, with same combinatorial type so that the corresponding conjugacies $\phi_{j, \ell}$ does not converge in an uniform way to identity: in other words we can select $\epsilon>0$ so that $\left|\phi_{j_{i}, \ell_{i}}(z)-z\right| \geq \epsilon$, for some $z \in \mathbb{C}$ and with $j_{i} \rightarrow \infty$. But lemma 7.3 and proposition 7.4 say that a subsequence of $\phi_{j_{i}, \ell_{i}}$ converges to a conjugacy between two bi-infinite towers, which do not support invariant line fields, so this conjugacy is a conformal map, hence it is the identity, which is a contradiction. Since $f \in \mathcal{P}_{n}^{\infty}\left(C_{1}, C\left(C_{1}\right)\right)$, we can select representations $R^{k}(f): U^{k} \rightarrow V^{k}$ which belongs to $\mathcal{P}_{n}^{\infty}\left(C_{1}, \tilde{C}\left(C_{1}\right)\right)$ and furthermore they are restrictions of iterates of $f$. This is possible for $k \geq k_{0}\left(C_{1}\right)$. Then $R^{k}(g): \tilde{U}^{k} \rightarrow \tilde{V}^{k}$, where $\tilde{U}^{k}=\phi\left(U^{k}\right)$ and $\tilde{V}^{k}=\phi\left(V^{k}\right)$, is a representation of $R^{k}(g)$. Since $2 \delta-K\left(R^{k}(f)\right) \subset V^{k}$, for some $\delta=\delta\left(C_{1}\right)$, and $\phi_{j}$ is close to identity, one gets $\delta-K\left(R^{k}(g)\right) \subset \tilde{V}^{k}$ for $k \geq$ $k_{1}\left(C_{1}, C_{2}\right)$. By lemmas 4.1 and $4.4, R^{k}(g) \in \mathcal{P}_{n}^{\infty}\left(C_{1}, C_{3}\left(C_{1}\right)\right)$, which proves the first statement. To prove the second one, note that $\delta-K\left(R^{k}(f)\right) \subset \tilde{V}^{k}$ and $(\delta / 2)$ $K\left(R^{k}(g)\right) \subset \delta-K\left(R^{k}(f)\right)$, for $k \geq k_{2}\left(C_{2}\right)$. By corollary $4.3 R^{k}(g): R^{k}(g)^{-n}\left(\tilde{V}^{k}\right) \rightarrow$ $R^{k}(g)^{-n+1}\left(\tilde{V}^{k}\right)$ is a representation in $B(\delta-K(f))$, where $n=n\left(\delta / 2, C_{3}\right)$. Since $\phi_{j}$ converges to identity, uniformly in compact sets, and the diameter of $\delta-K\left(R^{k}(f)\right)$, after the normalization $R^{k}(f)(0)=1$, is bounded, the proof is finished. 
7.2. Exponential Contraction. The exponential contraction of the renormalization operator in the hybrid classes of the infinitely renormalizable quadratic-like maps with bounded combinatorics was proved by C. McMullen ([McM2]). Indeed, McMullen's argument is quite general, and it was successfully applied for certain results about Kleinian groups ([McM2] and critical circle maps $([\mathrm{dFdM}])$. Let $\mathbb{H}^{3}$ be the hyperbolic space and identify the Riemann sphere $\mathbb{S}^{2}$ with its ideal boundary. If $K$ is a subset of the Riemann sphere, denote by hull $(K)$ the convex hull of the set of points in $\mathbb{H}^{3}$ contained in geodesics which arrive in both directions in a point of $K$. Furthermore, given a quasiconformal vector field $v$ in $\mathbb{S}^{2}$, we said that $v$ is a quasiconformal deformation of a polynomial like map $f$ if $\bar{\partial} v$ is invariant by $f$. For a continuous vector $v$ in $\mathbb{S}^{2}$, define the visual distortion $M v: \mathbb{H}^{3} \rightarrow \mathbb{R}^{+}$by

$$
M v(p)=\inf _{\bar{\partial} w=0}\|v-w\|_{\infty}(p)
$$

Here $\|\cdot\|_{\infty}(p)$ denotes the visual metric at point $p$ (see [McM2]). The visual distortion measure the distance of $v$ of the conformal vector fields. For instance,

$$
\sup _{p \in \mathbb{H}^{3}} M v(p)
$$

is finite if and only if $v$ is a quasiconformal vector field on the Riemann sphere. For an introduction of quasiconformal vector fields, visual distortion and it properties, see [McM2]. The following result is the main tool to prove the Dynamic Inflexibility Theorem in our setting:

Main Lemma 7.1. For any $C_{1}, C_{2}$ there exists $r\left(C_{1}, C_{2}\right)$ with the following property: let $f \in \mathcal{P}_{n}^{\infty}\left(C_{1}, C_{2}\right)$ and let $v$ be a quasiconformal deformation of $f$. Furthermore assume $S(p, r) \subset$ hull $(K(f))$. Then

$$
M v(p) \leq \frac{1}{2} \sup _{q \in S(p, r)} M v(q)
$$

Proof. The proof will be exactly as in lemma 9.12 in [McM2], with some small modifications to avoid technical definitions: Suppose, by contradiction, there exist sequences $r_{i} \rightarrow \infty, v_{i}, p_{i} \in \mathbb{C}$ and $f_{i} \in \mathcal{P}_{n}^{\infty}\left(C_{1}, C_{2}\right)$ so that

- $S\left(p_{i}, r_{i}\right) \subset \operatorname{hull}\left(K\left(f_{i}\right)\right)$,

- $M v_{i}(p) \geq 1 / 2$,

- $\sup _{q \in S\left(p_{i}, r_{i}\right)} M v_{i}(q) \leq 1$.

We can assume that $p_{i}=p$. Then $S\left(p, r_{i}\right) \subset \operatorname{hull}\left(K\left(f_{i}\right)\right)$, with $r_{i} \rightarrow \infty$, which implies that, for all $z \in \overline{\mathbb{C}}$ and $\epsilon>0$, dist $t_{\overline{\mathbb{C}}}\left(z, K\left(f_{i}\right)\right) \leq \epsilon$, if $i$ is large enough. In particular, by small Julia sets everywhere theorem, for any $z \in \mathbb{C}$ and $d>0$ there exists a sequence of polynomial-like maps $g_{i}: U_{i} \rightarrow V_{i}$, for $i$ large enough, so that

- $g_{i} \in \mathcal{F}\left(f_{i}\right)$,

- $g_{i} \in \mathcal{P}_{n}(C), C=C\left(C_{1}, C_{2}\right)$,

- $\operatorname{diamK}\left(g_{i}\right) \sim d$,

- $\operatorname{dist}_{E u c l}\left(z, K\left(g_{i}\right)\right) \leq C d$.

Because $\sup _{q \in S\left(p, r_{i}\right)} M v_{i}(q) \leq 1$, with $r_{i} \rightarrow \infty$, we can assume that the sequence $v_{i}$, up to sums with conformal fields in the Riemann sphere, converge uniformly to a quasiconformal vector $v_{\infty}$. Moreover $\bar{\partial} v_{i} \rightarrow \bar{\partial} v_{\infty}$ as distributions. In particular $\mu_{\infty}=\bar{\partial} v_{\infty}$ is invariant by any limit of the sequence $g_{i}$. Since $d$ is arbitrary, we obtained polynomial-like maps close to each point in the complex plane, in any scale, which keeps $\mu_{\infty}$ invariant. We claim that $\mu_{\infty}=0$. Otherwise, select $z$ a point of almost continuity of $\mu_{\infty}$. Hence $\mu_{\infty}$ is almost a straight Beltrami field 
near to $z$, which is impossible since there are polynomial-like maps (which form a compact family after conjugacies by affine maps) in all scales so that $\mu_{\infty}$ is invariant for them. But this is a contradiction, since $M v_{i}(p) \geq 1 / 2 \operatorname{implies} M v_{\infty}(p) \geq 1 / 2$, so $\mu_{\infty} \neq 0$.

Theorem 4 (Exponential contraction). Let $f \in \mathcal{A}_{n}^{\infty}\left(C_{1}\right)$ and let $g$ be a map in $\mathcal{P}_{n}^{\infty}\left(C_{1}, C_{2}\right)$ with the same combinatorics that $f$. There exist $k_{0}=k_{0}(g)$ and $\delta$ so that $R^{k}(g) \in B(\delta-K(f))$, for $k \geq k_{0}$ and furthermore

$$
\left|R^{k}(f)(z)-R^{k}(g)(z)\right| \leq \alpha^{k}
$$

for $z \in \delta-K(f)$ and $\alpha<1$. Here $\delta$ and $\alpha$ depends only on $C_{1}$.

Proof. By the dynamic inflexibility theorem ([McM2]), if $\phi: \mathbb{C} \rightarrow \mathbb{C}$ is a $K$-quasiconformal conjugacy between $f: U_{f} \rightarrow V_{f}$ and $g: U_{g} \rightarrow V_{g}, f, g \in \mathcal{P}_{n}^{\infty}\left(C_{1}, C_{2}\right)$, with $\phi\left(U_{f}\right)=$ $U_{g}$ then $\phi$ is $C^{1+\beta}$-conformal at 0 . It is not difficult to verify in the proof of dynamic inflexibility theorem that

$$
\beta=\beta\left(K, r\left(C_{1}, C_{2}\right), \delta\left(C_{1}, C_{2}\right)\right)
$$

Here $r$ is as in the previous lemma. Note that we can select $\phi$ such that $K=$ $K\left(C_{1}, C_{2}\right)$. Hence $\phi$ satisfies

$$
\phi(x)=\phi^{\prime}(0) \cdot x+O\left(|x|^{1+\beta}\right)
$$

Since diam $K\left(R^{k}(f)\right) \leq C \cdot \lambda^{k}$ for some $C>0$ and $\lambda<1$, after normalize $R^{k}(f)$ so that $R^{k}(f)(0)=1=R^{k}(g)(0)$, $\phi$ define a conjugacy $\phi_{k}$ satisfying

$$
\phi_{k}(x)=x+O\left(\alpha^{k}\right)
$$

for some $\alpha<1$. Using arguments as in theorem 3, the proof is finished.

\section{Appendix}

8.1. A fixed point theorem. The following fixed point theorem was proved by de Melo and van Strien $([\mathrm{dMvS}])$ when $K$ is a simplex.

Proposition 8.1 (de Melo-van Strien fixed point theorem [dMvS]). Let $K$ be $a$ bounded closed convex body in a finite dimensional normed linear space and let $T:$ int $K \rightarrow$ int $K$ be a continuous function such that

$$
\lim _{x \rightarrow \partial K} \frac{|T(x)-x|}{\operatorname{dist}(x, \partial K)}=\infty
$$

Then $T$ has a fixed point in int $K$.

The proof of de Melo-van Strien can be generalized to convex bodies using the following result: Let $K_{1}$ and $K_{2}$ be two bounded closed convex bodies in a finite dimensional normed linear space. The radial projection $\phi: K_{1} \rightarrow K_{2}$ is defined by (1) $\phi(0)=(0)(2)$ If $x_{i}, i=1,2$ are the unique points such that a ray beginning at 0 crosses $\partial K_{i}$ then $\phi\left(\lambda x_{1}\right)=\lambda x_{2}$, for $\lambda>0$. Then

Lemma 8.1 (Sz.-Nagy\&Klee Theorem: [K]). The radial projection between $K_{1}$ and $K_{2}$ is bi-Lipschtizian. 
8.2. Hyperbolic domains on the plane. Let $\Omega$ be a hyperbolic domain on the plane and $\rho_{\Omega}|d z|$ its hyperbolic metric. For $z \in \Omega$, define

$$
\beta_{\Omega}(z)=\inf \left\{\left|\log \frac{|z-a|}{|b-a|}\right|: a, b \in \partial \Omega ;|z-a|=\operatorname{dist}(z, \partial \Omega)\right\}
$$

To compare the Euclidean and hyperbolic metric on $\Omega$, we will use the following Beardon-Pommerenke results:

Proposition $8.2([\mathrm{BP}])$. There exists a constant $C$, with does not depend on $\Omega$, such that

$$
\frac{1}{2 \sqrt{2}} \frac{1}{\operatorname{dist}(z, \partial \Omega)} \leq \rho_{\Omega}(z) \leq \frac{1}{\operatorname{dist}(z, \partial \Omega)} \frac{C+\pi / 4}{C+\beta_{\Omega}(z)}
$$

In this proposition and in the following lines, $\operatorname{dist}(x, y)$ and $\operatorname{diam}(A)$ are distances and diameters with respect to the Euclidean metric on $\mathbb{C}$, and, if $M$ is a hyperbolic planar domain, then $\operatorname{dist}_{M}(x, y)$ and $\operatorname{diam}_{M}(A)$ are distances and diameters with respect the hyperbolic metric of $M$. An annulus $A \subset \Omega$ is essential if the bounded component of $\mathbb{C} \backslash A$ contain points in $\partial \Omega$.

Corollary $8.1([\mathrm{BP}])$. The following holds:

- If $\Omega$ is a hyperbolic domain whose any essential annulus has modulus bounded by $M$, then there exists $C(M)$ such that

$$
\frac{1}{C} \frac{1}{\operatorname{dist}(z, \partial \Omega)} \leq \rho_{\Omega}(z)<C \frac{1}{\operatorname{dist}(z, \partial \Omega)}
$$

For all $z \in \Omega$;

- If (16) holds, then there exist $M(C)$ such that any essential annulus has modulus bounded by $M$.

The domains satisfying the hypothesis of the previous corollary will be called $M$-uniform domains. Observe that if $\Omega$ is a uniform domain with maximum essential modulus bounded by $M$ and $D$ is a simply connected region in the plane, then $D \cap \Omega$ is also a uniform domain with the same bound for the maximal essential modulus.

Proposition 8.3. Let $\gamma$ be a Jordan curve in a $M$-uniform domain $U$ with length $\ell \leq \ell_{1}$ in the hyperbolic metric of $U$ and let $D$ be the bounded region in $\mathbb{C}-\gamma$. Then

$$
C_{1}\left(M, \ell_{1}\right) \operatorname{diam}(D \cap \partial U) \leq \operatorname{dist}(\gamma, D \cap \partial U) \leq C_{2}(M) \operatorname{diam}(D \cap \partial U)
$$

Moreover

$$
\operatorname{diam}(\gamma) \leq C_{2}\left(\ell_{1}, M\right) \operatorname{diam}(D \cap \partial U)
$$

Proof. Note that

$$
\operatorname{dist}(\gamma, D \cap \partial U) \leq C_{2}(M) \operatorname{diam}(D \cap \partial U)
$$

otherwise there will be a large essential ring in $U$. Denote $d=\left(1+C_{1}\right) \operatorname{diam}(D \cap \partial U)$ and fix $\lambda>1$. Select an arbitrary $z \in D \cap \partial U$ and define

$$
A_{n}=\left\{x \in \mathbb{C}: \lambda^{n} d \leq \operatorname{dist}(x, z) \leq \lambda^{n+1} d\right\}
$$

Let $\alpha \subset U \cap A_{n}, n \geq 1$, be a curve which touch both components of $\partial A_{n}$. Then the Euclidean length of $\alpha$ is at least $\lambda^{n}(\lambda-1) d$ and, if $\rho|d z|$ is the hyperbolic metric on $U$ then

$$
\ell \geq \int_{0}^{\lambda^{n}(\lambda-1) d} \rho(\gamma(t))\left|\gamma^{\prime}(t)\right| d t \geq C(M) \int_{0}^{\lambda^{n}(\lambda-1) d} \frac{1}{\operatorname{dist}(\gamma(t), D \cap \partial U)} d t \geq\left(1-\frac{1}{\lambda}\right) d
$$


which proves the lemma. If the diameter of $\gamma$ is large relative to diameter of $D \cap \partial U$ then $\gamma$ crosses many rings $A_{n}$, so its hyperbolic length is large, which is impossible. To obtain the lower bound to $\operatorname{dist}(\gamma, D \cap \partial U)$, notice that

$$
\gamma \subset \bigcup_{i \leq N} B\left(x_{i}, \frac{\operatorname{dist}\left(x_{i}, \partial \Omega\right)}{2}\right)
$$

for some $x_{i}$ in $\gamma$ and $N=N\left(\ell_{1}, M\right)$. It is easy to see that

$$
\operatorname{diam}(\gamma) \leq C(N) \operatorname{dist}\left(x_{i}, \partial \Omega\right),
$$

for any i. Since $\operatorname{diam}(\gamma) \geq \operatorname{diam}(\partial \Omega \cap U)$, the proof is complete.

Remark 8.1. The previous lemma will be used in the following situation: Let $f: U \rightarrow V$ be an infinitely renormalizable polynomial-like map of type $n$ with bounded combinatorics. Then the postcritical set $P$ is a Cantor set with bounded geometry and hence $V-P$ is a $M$-uniform domain. Furthermore, the hyperbolic length of the closed geodesics in $V-P$ is under control. Thus we can apply lemma 8.3 for these geodesics.

The following proposition is a kind of Koebe lemma for $M$-uniform domains: the proof is easy.

Proposition 8.4. Let $f: U \rightarrow \Omega_{2}$ be a covering map so that

- $\Omega_{2}$ is a $M$-uniform domain;

- The domain $U$ is contained in a $M$-uniform domain $\Omega_{1}$.

If $\tilde{A}$ is a simply connected domain inside $\Omega_{2}$ and $A$ is a connected component of $f^{-1}(\tilde{A})$, then

and

$$
\frac{1}{C} \leq \frac{\left|f^{\prime}\left(z_{1}\right)\right|}{\left|f^{\prime}\left(z_{2}\right)\right|} \leq C
$$

$$
\frac{1}{C} \leq \frac{\left\|f^{\prime}\left(z_{1}\right)\right\|_{\Omega_{1}, \Omega_{2}}}{\left\|f^{\prime}\left(z_{2}\right)\right\|_{\Omega_{1}, \Omega_{2}}} \leq C
$$

for $z_{1}, z_{2} \in A$ and $C=C\left(M, \operatorname{diam}_{\Omega_{2}}(\tilde{A})\right)$.

8.3. Quasiconformal mappings. We say that a Jordan curve $J \subset \mathbb{C}$ is a Cquasicircle if there is a $C$-quasiconformal map $\phi$ on the Riemann Sphere such that $\phi\left(S^{1}\right)=C$.

Lemma 8.2. Let $\psi: \mathbb{D} \rightarrow \mathbb{D}$ be a $C_{1}$-quasiconformal map and $x, y \in \mathbb{D}$ with dist $_{\mathbb{D}}(x, y) \leq D$. Then there exists a $C_{2}\left(C_{1}, D\right)$-quasiconformal map $\tilde{\psi}: \mathbb{D} \rightarrow \mathbb{D}$ which coincide with $\psi$ in a neighborhood of $S^{1}$ and $\tilde{\psi}(x)=y$.

Proof. It follows of lemma 5.2.3 in [GS] or the moving lemma at pg. 288 in [L1].

Proposition 8.5. Let $J$ be a $C_{1}$-quasicircle and $x$ a point in the bounded domain in $\mathbb{C}-J$ such that $\operatorname{dist}(x, J) \geq \epsilon \operatorname{diam}(J)$. Then there exists a $C_{2}\left(C_{1}, \delta\right)$ quasiconformal map on the plane $\tilde{\phi}$ such that $\tilde{\phi}\left(S^{1}\right)=J$ and $\tilde{\phi}(0)=x$.

Proof. Assume, without loss of generality, that diam $J=1$. Consider a $C_{1}$ - quasiconformal map in $\overline{\mathbb{C}}$ such that $\phi\left(S^{1}\right)=J$. After a composition with a Moebius transformation which preserves the circle, we can assume that $\phi(\infty)=\infty$. Furthermore, after translate and rotate $J$, we can assume that $\phi(0)=0$ and $\phi(1)=1$. Since the set of $C_{1}$-quasiconformal maps on the plane such that $\phi(0)=0$ and 
$\phi(1)=1$ is compact, there exists $\delta>0$ such that for $a, b \in \bar{D},|a-b| \leq \delta$ implies $|\phi(a)-\phi(b)| \leq \epsilon$. In particular $\operatorname{dist}\left(\phi^{-1}(x), S^{1}\right) \geq \delta$. By the previous lemma, we obtain a $C_{2}(C, \delta)$-quasiconformal map $\tilde{\phi}$ on the plane which is equal to $\phi$ outside $\mathbb{D}$ and $\tilde{\phi}(0)=x$.

\section{REFERENCES}

[BP] A. F. Beardon and Ch. Pommerenke. The Poincaré metric of plane domains. J. London Math. Soc. (2), 18:475-483, 1978.

[BL] A. Blokh and M. Lyubich Measure and dimension of solenoidal attractors of onedimensional dynamical systems. Comm. Math. Phys., 127:573-583.

[BH] B. Branner and J. Hubbard. The iteration of cubic polynomials. I. the global topology of parameter space. Acta Math., 160:143-206, 1998.

[DH] A. Douady and J. Hubbard. On the dynamics of polynomial-like mappings. Ann. Sci. École Norm. Sup. (4), 18:287-343, 1985.

[dFdM $]$ E. de Faria and W. de Melo. Rigidity of critical circle mappings II. J. Am. Math. Soc. , 13:343-370, 2000 .

[FS] J. Fornaess and N. Sibony. Random iterations of rational functions. Ergod. Th. \&3 Dynam. Sys., 11:687-708, 1991.

[GS] J. Graczyk and G. Światek. The Real Fatou Conjecture, volume 144 of Annals of Mathematics Studies. Princeton Univ. Press, Princeton, New Jersey, 1998.

[H1] J. Hu. Renormalization, rigidity and universality in bifurcation theory. PhD thesis, City University of New York, 1995.

[H2] J. Hu. Bounded geometry in the supports of ergodic invariant probability measures. Internat. J. Bifur. Chaos Appl. Sci. Engrg., 8:1957-1973, 1998.

[K] Jr. Klee, V. L. On a theorem of Béla Sz.-Nagy. Amer. Math. Monthly, 60:618-619, 1953.

[L1] M. Lyubich. Dynamics of quadratic polynomials. I, II. Acta Math, 178:185-297, 1997.

[L2] M. Lyubich. Feigenbaum-Coullet-Tresser universality and Milnor's hairiness conjecture. Ann. of Math.(2), 149:319-420, 1999.

[McKZ] R. MacKay and J. van Zeijts. Period doubling for bimodal maps: a horseshoe for a renormalisation operator. Nonlinearity, 1:253-277, 1988.

[McM1] C. McMullen. Complex dynamics and renormalization, volume 135 of Annals of Mathematics Studies. Princeton University Press, Princeton, NJ, 1994.

[McM2] C. McMullen. Renormalization and 3-manifolds which fiber over the circle, volume 142 of Annals of Mathematics Studies. Princeton University Press, Princeton, NJ, 1996.

[McMS] C. McMullen and D. Sullivan. Quasiconformal homeomorphisms and dynamics. III. the Teichmuller space of a holomorphic dynamical system. Adv. Math., 135:351-395, 1998.

[dMvS] W. de Melo and S. van Strien. One-dimensional Dynamics. Springer-Verlag, New York, 1993.

[PR] F. Przytycki and S. Rohde. Rigidity of holomorphic Collet-Eckmann repellers. Ark. Mat., 37:357-371, 1999.

[Sh1] W. Shen. On the measurable dynamics of real rational functions. Ergodic Theory Dynam. Systems 23:957-983, 2003.

[Sh2] W. Shen. On the metric properties of multimodal interval maps and $C^{2}$ density of Axiom A. Invent. Math. 156:301-403, 2004.

[Sm1] D. Smania. Complex bounds for multimodal maps: bounded combinatorics. Nonlinearity, 14:1311-1330, 2001.

[Sm2] D. Smania. Renormalization theory for multimodal maps. Ph. D. Thesis, IMPA, Feb/2001.

[SN] S. van Strien and T. Nowicki. Polynomial maps with a Julia set of positive Lebesgue measure: Fibonacci maps. unpublished, 1994.

[Su] D. Sullivan. Bounds, quadratic differentials, and renormalization conjectures. In AMS Centennial Publications: Mathematics into Twenty-first Century, volume 2. AMS, 1992. 
IMPA, Rio DE JANEIRO, BRAZIL

Current address: Departamento de Matemática, ICMC-USP-Campus de São Carlos, Caixa Postal 668, São Carlos-SP, CEP 13560-970, Brazil.

E-mail address: smania@icmc.usp.br

$U R L$ : www.icmc.usp.br/ smania/ 